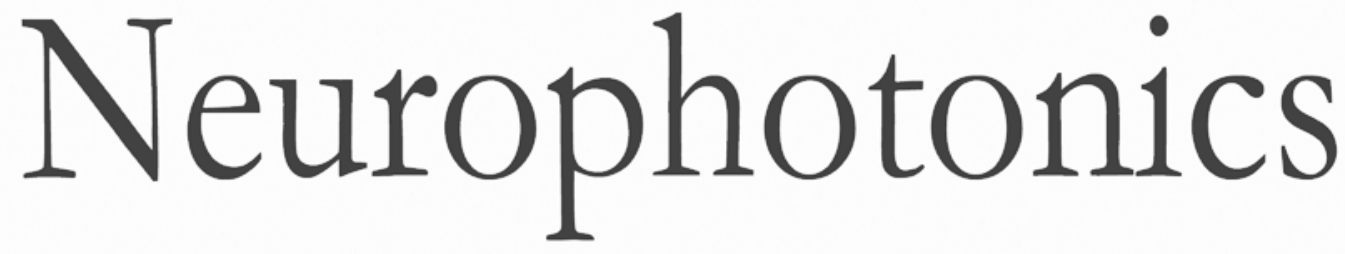

\title{
Noninvasive continuous optical monitoring of absolute cerebral blood flow in critically ill adults
}

Lian $\mathrm{He}$

Wesley B. Baker

Daniel Milej

Venkaiah C. Kavuri

Rickson C. Mesquita

David R. Busch

Kenneth Abramson

Jane Y. Jiang

Mamadou Diop

Keith St. Lawrence

Olivia Amendolia

Francis Quattrone

Ramani Balu

W. Andrew Kofke

Arjun G. Yodh 


\title{
Noninvasive continuous optical monitoring of absolute cerebral blood flow in critically ill adults
}

\author{
Lian He, ${ }^{\mathrm{a}, \star}$ Wesley B. Baker, ${ }^{\mathrm{a}, \mathrm{b}}$ Daniel Milej, ${ }^{\mathrm{c}, \mathrm{d}}$ Venkaiah C. Kavuri, ${ }^{\mathrm{a}}$ Rickson C. Mesquita, ${ }^{e}$ David R. Busch, \\ Kenneth Abramson, ${ }^{a}$ Jane Y. Jiang, ${ }^{a}$ Mamadou Diop, ${ }^{c, d}$ Keith St. Lawrence, ${ }^{c, d}$ Olivia Amendolia, ${ }^{\text {h }}$ \\ Francis Quattrone, ${ }^{\mathrm{h}}$ Ramani Balu, ${ }^{\mathrm{h}, \mathrm{i}}$ W. Andrew Kofke, ${ }^{\mathrm{b}, \mathrm{h}}$ and Arjun G. Yodh ${ }^{\mathrm{a}, \mathrm{b}}$ \\ aUniversity of Pennsylvania, Department of Physics and Astronomy, Philadelphia, Pennsylvania, United States \\ bUniversity of Pennsylvania, Department of Anesthesiology and Critical Care, Perelman School of Medicine, Philadelphia, \\ Pennsylvania, United States \\ ${ }^{c}$ Western University, Department of Medical Biophysics, London, Ontario, Canada \\ 'Lawson Health Research Institute, Imaging Division, London, Ontario, Canada \\ eUniversity of Campinas, Institute of Physics, Campinas, São Paulo, Brazil \\ fUniversity of Texas Southwestern, Department of Neurology and Neurotherapeutics, Dallas, Texas, United States \\ gUniversity of Texas Southwestern, Department of Anesthesiology and Pain Management, Dallas, Texas, United States \\ hUniversity of Pennsylvania, Department of Neurosurgery, Perelman School of Medicine, Philadelphia, Pennsylvania, United States \\ 'University of Pennsylvania, Department of Neurology, Perelman School of Medicine, Philadelphia, Pennsylvania, United States
}

\begin{abstract}
We investigate a scheme for noninvasive continuous monitoring of absolute cerebral blood flow (CBF) in adult human patients based on a combination of time-resolved dynamic contrast-enhanced near-infrared spectroscopy (DCE-NIRS) and diffuse correlation spectroscopy (DCS) with semi-infinite head model of photon propogation. Continuous CBF is obtained via calibration of the DCS blood flow index (BFI) with absolute CBF obtained by intermittent intravenous injections of the optical contrast agent indocyanine green. A calibration coefficient $(\gamma)$ for the CBF is thus determined, permitting conversion of DCS BFI to absolute blood flow units at all other times. A study of patients with acute brain injury $(N=7)$ is carried out to ascertain the stability of $\gamma$. The patientaveraged DCS calibration coefficient across multiple monitoring days and multiple patients was determined, and good agreement between the two calibration coefficients measured at different times during single monitoring days was found. The patient-averaged calibration coefficient of $1.24 \times 10^{9}(\mathrm{~mL} / 100 \mathrm{~g} / \mathrm{min}) /\left(\mathrm{cm}^{2} / \mathrm{s}\right)$ was applied to previously measured DCS BFI from similar brain-injured patients; in this case, absolute CBF was underestimated compared with $\mathrm{XeCT}$, an effect we show is primarily due to use of semi-infinite homogeneous models of the head. (C) The Authors. Published by SPIE under a Creative Commons Attribution 3.0 Unported License. Distribution or reproduction of this work in whole or in part requires full attribution of the original publication, including its DOI. [DOI: 10.1117/1.NPh.5.4.045006]
\end{abstract}

Keywords: diffuse correlation spectroscopy; time-resolved contrast-enhanced near-infrared spectroscopy; absolute cerebral blood flow; brain-injured patients; continuous monitoring.

Paper 18042R received Jul. 21, 2018; accepted for publication Oct. 29, 2018; published online Nov. $23,2018$.

\section{Introduction}

Brain-injured patients are prone to development of secondary brain injury during the management period, and therapeutic interventions for secondary brain injury hold potential for meaningful impact on long-term patient outcome. ${ }^{1}$ More specifically, clinical studies suggest that brain-injured patients with impaired cerebral autoregulation are unusually susceptible to secondary ischemic insult ${ }^{2,3}$ and that when cerebral blood flow $(\mathrm{CBF})$ drops below a critical threshold, the development of irreversible tissue damage can occur; ${ }^{4}$ whereas if CBF exceeds the brain's metabolic requirement, unregulated hyperemia can cause brain edema. ${ }^{5-7}$ Thus, CBF monitoring during the management period can improve acute care via rapid detection and resultant therapy to attenuate conditions that can lead to secondary brain injury. ${ }^{1}$

To date, several measurement methods, including PET, MRI $,{ }^{9} \mathrm{Xe}^{133},{ }^{10} \mathrm{Xe}-\mathrm{CT},{ }^{11,12}$ transcranial Doppler, ${ }^{13,14}$ thermal diffusion, ${ }^{15}$ jugular bulb oximetry, ${ }^{16}$ and $\mathrm{EEG}^{17,18}$ have been utilized to infer or to directly evaluate $\mathrm{CBF}$, but none of these offers truly continuous noninvasive absolute CBF monitoring capabilities at the bedside. The present study explores the potential of a combination of near-infrared (NIR) optical methods for

*Address all correspondence to: Lian He, E-mail: lianh@sas.upenn.edu noninvasive continuous bedside monitoring of absolute CBF in a cohort of adult human patients with brain injury. Thus, patient treatment needs not be interrupted, and risks of secondary insults from patient transport (e.g., to MRI, PET instruments) are reduced. The approach uses time-resolved dynamic contrast-enhanced near-infrared spectroscopy (DCE-NIRS) to calibrate the blood flow index (BFI) determined by diffuse correlation spectroscopy (DCS); similar brain volumes are sampled by the two techniques, and the same light transport model is used for data analysis and generation of DCS calibration coefficients. The calibrated BFI subsequently permits continuous bedside monitoring of $\mathrm{CBF}$ in physiological units, e.g., $\mathrm{mL}$ of blood/100 g/ min. ${ }^{19,20}$ In this contribution, to establish the repeatability of the calibration coefficients, we cross calibrate these two quantitatively different and complementary NIR optical blood flow (BF) measurement techniques at different time points during the care of a cohort of patients with acute brain injury. We also determine an average calibration coefficient based on data across multiple monitoring days and multiple patients. Finally, we apply this calibration coefficient to assess flow in critically ill patients with subarachnoid hemorrhage from a previous study carried out in our groups. ${ }^{21}$

DCS employs NIR light to directly and noninvasively measure local microvascular $\mathrm{CBF}^{22-26}$ This optical technique is 
suitable for continuous bedside monitoring; it has excellent temporal resolution, ${ }^{27}$ offers penetration into cerebral tissues through intact scalp and skull, ${ }^{28}$ and is compatible for combined use with traditional NIRS devices. ${ }^{21,25,29-31}$ To date, DCSmeasured relative $\mathrm{BF}$ changes have been extensively validated in many tissues and against numerous other techniques, ${ }^{21,23}$ but the DCS BFI has unusual units $\left(\mathrm{cm}^{2} / \mathrm{s}\right)$. Absolute calibration of DCS in traditional units $(\mathrm{mL} / 100 \mathrm{~g} / \mathrm{min})$ would enable other useful CBF assessments of the physiological condition of braininjured patients, e.g., at baseline and via patient comparison. Recently, a DCE-NIRS method that employs injection of a light-absorbing optical contrast agent, indocyanine green (ICG), was demonstrated to measure absolute $\mathrm{CBF}^{19,32-34}$ Specifically, the time-dependent absorption signal of an ICG bolus through cerebral tissue enables measurement of absolute $\mathrm{CBF}$ at a single time point. This approach was applied to measure CBF in piglets and adult pigs, ${ }^{20,32,35}$ newborn infants, ${ }^{36,37}$ and healthy adults. ${ }^{34,38,39}$ Notably, in a piglet study, comparisons of DCE-NIRS and CT perfusion measurements showed good agreement. $^{32}$ Unfortunately, absolute CBF measurement by DCE-NIRS requires injection of an ICG bolus and is also limited by a maximum recommended daily dose; both factors are barriers to continuous CBF monitoring.

Here, we investigate a scheme for noninvasive continuous bedside monitoring of absolute CBF in adult human brain-injured patients based on a combination of time-resolved DCE-NIRS and DCS. By comparing DCE-NIRS to concurrent DCS measurements, a calibration coefficient for the $\mathrm{CBF}$ is determined that permits conversion of DCS BFI into absolute BF units at other times during injury management. The concurrent measurements enabled assessment of the stability of ICG calibration coefficients obtained from each patient across single monitoring days. We found excellent agreement between the two calibration coefficients across single monitoring days. Additionally, we determined a patient-averaged calibration coefficient for DCS monitoring of absolute CBF. Significant correlation between CBF obtained with two optical techniques was also observed. Finally, the population-averaged calibration coefficient was applied to a group of adult brain-injured patients studied previously by our group, ${ }^{21}$ thereby taking first steps toward using DCS directly as a measure of absolute CBF. In total, the research suggests that the combined DCS and DCE-NIRS method should enable improved continuous, noninvasive, and quantitative DCS monitoring of absolute $\mathrm{CBF}$ at the bedside in the clinic.

\section{Methods}

\subsection{Dynamic Contrast-Enhanced Time-Resolved Near-Infrared Spectroscopy}

The DCE-NIRS technique treats the brain vascular bed as a linear, time-invariant system with single entrance and exit. $\mathrm{CBF}$ can be measured using DCE-NIRS by tracking the concentration of a NIR tracer in the brain vascular bed. ${ }^{32,40,41}$ Specifically, when a bolus of ICG-tracer is injected intravenously in a peripheral or central vein, then the rate of delivery of ICG to the brain vascular bed at time $t$ is $\mathrm{CBF} \cdot C_{a}(t)$, where $C_{a}(t)\left[\mu \mathrm{mol} \cdot L^{-1}\right]$ is the cerebral arterial ICG concentration (per volume of blood). If the amount of ICG in brain tissue varies linearly with respect to the arrival rate, and if $\mathrm{CBF}$ is constant in time (i.e., during the measurement), then the accumulated ICG concentration (per volume of tissue) in the brain tissue at time $t$, i.e., $Q(t)$ $\left[\mu \mathrm{mol} \cdot L^{-1}\right]$ is ${ }^{32,33}$
$Q(t)=\mathrm{CBF} \cdot C_{a}(t) * R(t)$,

where $*$ is the convolution operator, and $R(t)$ is the so-called impulse residue function, which can be viewed as the fraction of ICG remaining in cerebral vascular bed at time $t$ following an instantaneous ICG bolus into the arterial input at time zero, i.e., $C_{a, 0} \delta(t)$. Deconvolution with respect to $Q(t)$ and $C_{a}(t)$ permits $\mathrm{CBF} \cdot R(t)$ to be extracted. ${ }^{33}$ The magnitude of $\mathrm{CBF} \cdot R(t)$ at the initial time gives absolute $\mathrm{CBF}[\mathrm{mL} / 100 \mathrm{~g} / \mathrm{min}]$ as $R(0)=1$. (Note, a cerebral tissue density of $1.05 \mathrm{~g} / \mathrm{mL}$ was used to convert the CBF units of $\mathrm{mL} / 100 \mathrm{~mL} / \mathrm{min}$ obtained from the deconvolution to $\mathrm{mL} / 100 \mathrm{~g} / \mathrm{min}$.)

We used time-resolved NIRS (TR-NIRS) to measure $Q(t)$ on the forehead superior to the frontal sinuses [Fig. 1(a) and Sec. 2.1.1]. The width of $Q(t)$ is a metric of how quickly the ICG bolus passes through the TR-NIRS sampled tissue volume [Fig. 1(c)]. Note, a previous study demonstrated high correlation (slope not significantly different from unity) between $C_{a}(t)$ measured noninvasively with a dye densitometer attached to the finger and measured invasively through the radial artery. ${ }^{42}$ Assuming that the ICG concentration in the radial artery and the cerebral arteries have the same temporal shape $C_{a}(t)$ is approximated by the arterial ICG concentration in the finger, i.e., $C_{\text {finger }}(t)$ measured with a custom dye densitometer [Fig. 1(c), Sec. 6 Appendix 1]. The injected ICG bolus arrives at the cerebral arteries earlier than it arrives in the finger arteries. Therefore, the arterial ICG bolus transit time-delay between the finger arteries and the cerebral arteries, i.e., $t_{a}$ is a fitting parameter in the deconvolution of Eq. (1), and the experimentally derived deconvolution [e.g., Fig. 1(d)] is CBF $\cdot R\left(t-t_{a}\right)$. The shape of $C_{a}(t)$ was assumed to be similar to the shape of $C_{\text {finger }}(t)$, i.e., $C_{a}(t)=C_{\text {finger }}\left(t-t_{a}\right)$.

\subsubsection{Tissue ICG concentration measurement with TR-NIRS}

TR-NIRS delivers short NIR light pulses $(<100 \mathrm{ps})$ to a source position on the scalp; the pulse broadens as it propagates through tissue. At the detector, located a distance $r=3.2 \mathrm{~cm}$ away from the source on the scalp surface, the so-called temporal point spread function (TPSF) is measured, which is a histogram of the number of photons striking the detector as a function of the time difference, $\tilde{T}$, between the TR-NIRS source pulse trigger and the detection of photons [Fig. 1(b), histogram bin width $1 \mathrm{ps}$ ]. To track the kinetics of ICG passage through the sampled tissue volume following intravenous bolus injection, TPSFs were continuously acquired at a single wavelength $(808 \mathrm{~nm})$ at a sampling rate of $0.9 \mathrm{~Hz}$ (i.e., histogram integration time of $1.1 \mathrm{~s})$. Note that the time-domain photon diffusion equation models the distribution of photons as a function of photon time-of-flight through the tissue, i.e., $\phi(T)$, which is not identical to the experimentally measured TPSF because of instrument response function (IRF) effects. Using a semi-infinite homogeneous tissue model, the temporal cerebral tissue absorption changes caused by the transit of ICG through the brain can be determined via ${ }^{43}$

$\Delta \mu_{a}(t)=-\frac{2}{c\left(T_{2}^{2}-T_{1}^{2}\right)} \int_{T_{1}}^{T_{2}} \ln \frac{I_{t}(T)}{I_{0}(T)} \mathrm{d} T$.

Here $T \approx \tilde{T}-\tilde{T}_{0}$, where $\tilde{T}_{0}$ is the maximal photon count bin of the IRF measurement [Fig. 1(b), Sec. 2.3.2], $I_{0}(T)$ is the mean 
(a)

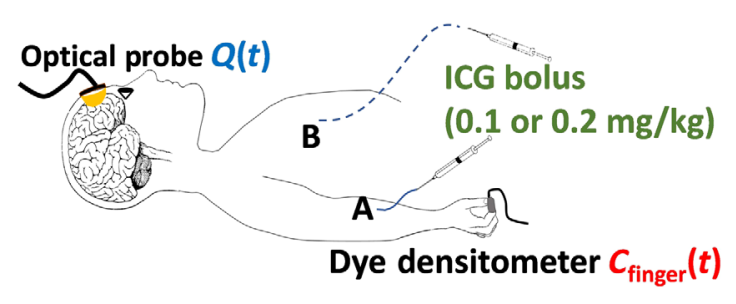

(c)

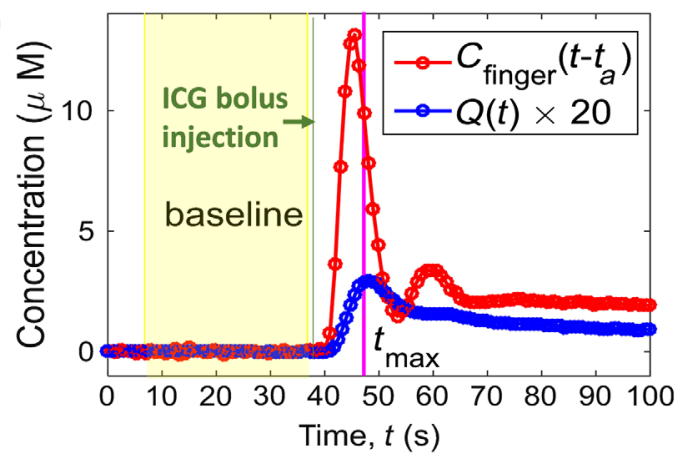

(b)
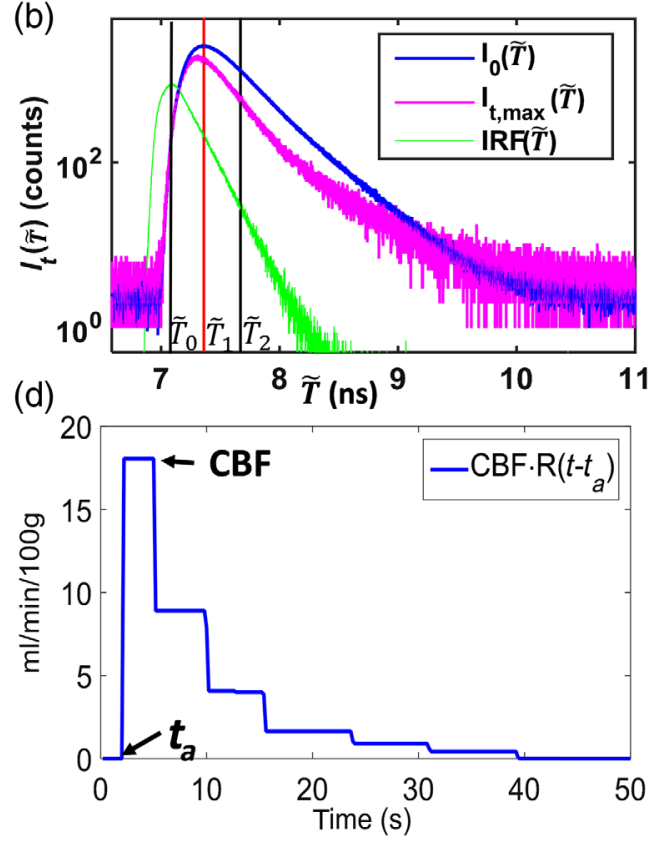

Fig. 1 (a) DCE-NIRS measurement of absolute CBF utilizes TR-NIRS to track the passage of an ICG bolus [rapidly injected $(<2 \mathrm{~s}$ ) in a peripheral (location A) or central vein (location $B$ )] through the tissue sampled by the optical probe. The average ICG concentration across the tissue volume, i.e., $Q(t)$, is measured by TR-NIRS (Sec. 2.1.1). The cerebral arterial ICG concentration is approximated using the arterial ICG concentration in the finger, i.e., $C_{\text {finger }}(t)$, which is measured with a customized dye densitometer (Sec. 6 and Appendix 1). (b) Exemplar effects on the TR-NIRS signals from a $0.2 \mathrm{mg} / \mathrm{kg}$ IV ICG bolus injection. Note, the TR-NIRS measurement is a histogram of the number of photons striking the detector as a function of the time difference, $\tilde{T}$, between the TR-NIRS source pulse trigger and the detected photons. The TR-NIRS measurement acquired at the time of maximal ICG concentration in the brain [i.e., $\left.I_{t, \max }(\tilde{T})\right]$ is attenuated relative to the baseline TR-NIRS measurement acquired prior to ICG injection [i.e., $I_{0}(\tilde{T})$ ]. Here, $\tilde{T}_{0}$ denotes the maximal photon count bin of the IRF, and $\tilde{T}_{1}$ and $\tilde{T}_{2}$ denote the histogram bins with maximal and half-maximal photon counts in the $I_{0}(\tilde{T})$ curve. (c) Temporal measurements of $Q(t)$ [via Eq. (2)] and $C_{\text {finger }}(t)$ in the same exemplar patient. The peak value of $Q(t)$ corresponds to an $0.065 \mathrm{~cm}^{-1}$ absorption change due to ICG. (d) The CBF-scaled impulse residue function, $R(t)$, retrieved from the deconvolution of the curves in (c). The height of the initial plateau yields CBF $(18 \mathrm{~mL} / \mathrm{min} / 100 \mathrm{~g})$. Here, $t_{a}(2 \mathrm{~s})$ is the arterial ICG bolus transit time delay between the finger arteries and the cerebral arteries, which is a fit parameter in the deconvolution.

TPSF acquired across the 30-s interval immediately prior to injection of the ICG bolus [Fig. 1(b)], $I_{t}(T)$ is the TPSF measured at time $t$ following ICG bolus injection, $c$ is the speed of light in tissue, and the limits $T_{1}$ and $T_{2}$ can be chosen to bound any time interval within the TPSF. In our study, $T_{1}$ was chosen to be the time-of-flight with maximal photon count at baseline, i.e., $I_{0}\left(T_{1}\right)=\max \left(I_{0}\right)$, and $T_{2}$ was the time for the baseline TPSF to fall to half the maximal photon count, i.e., $I_{0}\left(T_{2}\right) / I_{0}\left(T_{1}\right)=0.5 ; T_{2}>T_{1} . T_{1}$ and $T_{2}$ were chosen to roughly balance between increased brain sensitivity (long timeof-flights) and adequate signal-to-noise ratio (very long time-offlights have poor signal-to-noise ratio). Note, long timesof-flight are also more susceptible to fluorescence light. ${ }^{44}$ For $T_{1}<T<T_{2}$, Eq. (2) makes the approximation that $\ln \left[I_{t}(T) / I_{0}(T)\right] \approx \ln \left[\phi_{t}(T) / \phi_{0}(T)\right]$, where $\phi_{t}(T)$ and $\phi_{0}(T)$ denote the semi-infinite photon diffusion model computations of the photon distributions given the tissue absorption and scattering optical properties at time $t$ and baseline, respectively. Note, according to our computer simulations with varied IRF temporal width, about $10 \%$ underestimation in CBF should be expected when using Eq. (2) to analyze experimentally measured TPSF, i.e., compared with an ideal TPSF with no broadening of the laser pulse due to electro-optical components.
Moreover, we found that the CBF calculation is not very sensitive to the width of IRF [using Eq. (2)], i.e., $<5 \%$ difference between IRF full-width at half-maximum $($ FWHM $)=180$ versus 550 ps.

The ICG concentration in brain tissue is then calculated from the Beer-Lambert law, i.e.,

$Q(t)=\Delta \mu_{a}(t) /\left[\ln (10) \times \varepsilon_{\mathrm{ICG}}\right]$

where $\varepsilon_{\mathrm{ICG}}$ is the spectral extinction coefficient of ICG in plasma measured at $808 \mathrm{~nm}$ and $6.5 \mu \mathrm{M}$ concentration (191 OD/mM/cm ${ }^{45}$ ), and $\Delta \mu_{a}(t)$ is obtained via Eq. (2). Note that Eq. (3) assumes that changes in tissue absorption from chromophores other than ICG are negligible.

\subsection{Diffuse Correlation Spectroscopy}

DCS delivers highly coherent NIR light to a source position on the scalp, which then propagates diffusively inside the tissue. At a distance $r$ from the source, measured along the tissue surface, the rapid speckle intensity fluctuations of the multiply scattered light are measured. ${ }^{22,23,46-52}$ Specifically, these fluctuations, which are mainly induced by red blood cell motion, 
are quantified by the normalized intensity autocorrelation function, $g_{2}(r, \tau) \equiv\langle I(r, t) I(r, t+\tau)\rangle /\langle I(r, t)\rangle^{2}$, where $I(r, t)$ is the detected light intensity at time $t$ and distance $r$ from the source, $\tau$ is the delay-time, and the angle brackets, \langle\rangle , represent time-averages. The intensity autocorrelation function is related to the electric field autocorrelation function, $G_{1}(r, \tau) \equiv$ $\left\langle E^{*}(r, t) \cdot E(r, t)\right\rangle$, by the Siegert relation, $g_{2}(r, \tau)=1+$ $\beta\left|G_{1}(r, \tau) / G_{1}(r, 0)\right|^{2} \cdot{ }^{46,47} G_{1}(r, \tau)$ satisfies the correlation diffusion equation in highly scattering media, and $\beta$ is a constant that is inversely proportional to the number of speckles detected.

The tissue BFI and the parameter $\beta$ are extracted from the fit of the measured $g_{2}(\tau)$ to the homogeneous continuous-wave solution of the correlation diffusion equation in the semi-infinite geometry via the Siegert relation. (Limitations of the semi-infinite model are explored in Sec. 4.) The semi-infinite solution is

$G_{1}(r, \tau)=\frac{3 \mu_{s}^{\prime}}{4 \pi}\left\{\frac{\exp \left(-k_{D} r_{1}\right)}{r_{1}}-\frac{\exp \left(-k_{D} r_{2}\right)}{r_{2}}\right\}$,

where $\quad k_{D}^{2}=3 \mu_{s}^{\prime} \mu_{a}+6 \mu_{s}^{\prime 2} k_{0}^{2} \mathrm{BFI} \tau, \quad r_{1}=\sqrt{r^{2}+z_{0}^{2}}, \quad r_{2}=$ $\sqrt{r^{2}+\left(z_{0}+2 z_{b}\right)^{2}}, k_{0}=2 \pi n / \lambda, n$ is the tissue index of refraction (i.e., 1.4), $\lambda$ is the DCS light wavelength (i.e., $785 \mathrm{~nm}$ in the present study), $\mu_{a}$ is the tissue optical absorption coefficient, $\mu_{s}^{\prime}$ is the tissue reduced-scattering coefficient, $z_{0} \approx 1 /\left(\mu_{a}+\mu_{s}^{\prime}\right)$ is the effective depth of the source, $z_{b}=2 D\left(1+R_{\text {eff }}\right) /\left(1-R_{\text {eff }}\right)$ is the distance at which the fluence rate extrapolates to zero, $D=1 /\left[3\left(\mu_{a}+\mu_{s}^{\prime}\right)\right]$ is the diffusion constant, and $R_{\text {eff }}$ is an effective Fresnel reflection coefficient. For the present study, the index of refraction of the optical probe was approximately equivalent to the tissue, i.e., $R_{\text {eff }}=0$, and we concurrently measured $\mu_{a}$ and $\mu_{s}^{\prime}$ with TR-NIRS (see Sec. 7 and Appendix 2).

Although the DCS BFI has nontraditional units $\left(\mathrm{cm}^{2} / \mathrm{s}\right)$ for flow, BFI has been shown to be directly proportional to tissue $\mathrm{BF}$, i.e., $\mathrm{BF}=\gamma \cdot \mathrm{BFI}$, where $\gamma$ is a constant; this relationship has been observed by comparison with numerous independent methods. ${ }^{23,53} \gamma$ depends on the nature (e.g., arterial/capillary/ venule blood volume fractions) and geometry of the microvasculature underneath the probe, tissue heterogeneities underneath the probe, and tissue optical properties. The DCS calibration coefficient $\gamma$ for the brain (in our geometry) enables conversion of BFI to absolute CBF units, i.e.,

$\gamma=\mathrm{CBF}_{0} / \mathrm{BFI}_{0}$

where $\mathrm{CBF}_{0}$ and $\mathrm{BFI}_{0}$ are the $\mathrm{CBF}$ measured by DCE-NIRS and the BFI measured by DCS at the time of ICG bolus administration, respectively. The calibration coefficient $\gamma$ has units of $\left[\left(\mathrm{mL} \cdot 100 \mathrm{~g}^{-1} \cdot \mathrm{min}^{-1}\right) /\left(\mathrm{cm}^{2} / \mathrm{s}\right)\right]$. In practice, the DCS measurement was not acquired simultaneously with the DCE-NIRS measurement. Therefore, $\mathrm{BFI}_{0}$ at the time of ICG bolus administration was estimated from spline interpolation (implemented in MATLAB R2016a, Mathworks, Natick, Massachusetts) of the DCS BFI measurements made within the 10-minute interval encompassing the ICG bolus injection (Fig. 2). Alternatively, $\mathrm{BFI}_{0}$ can also be interpolated (with similar accuracy) by averaging two BFI measurements taken before and after ICG bolus injection. Absolute DCS BF is achieved by multiplying the measured BFIs and $\gamma$ at all other times during optical monitoring.

The primary goal of this clinical study is to determine and characterize $\gamma$ in critically brain-injured adult patients using independent measurements of CBF with DCE-NIRS at multiple points in time. A secondary goal is to investigate the accuracy

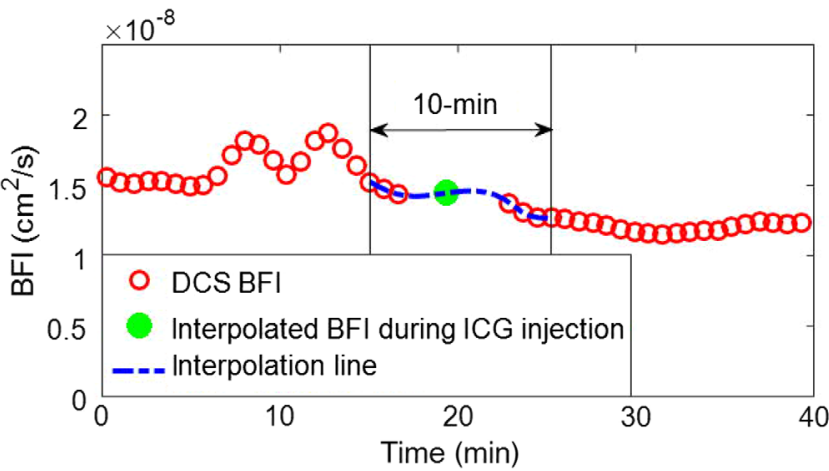

Fig. 2 An example showing the use of spline interpolation to estimate $\mathrm{BFI}_{0}$, i.e., the BFI at the time of ICG bolus injection. The red circles are continuously measured BFIs prior to and after the ICG injection. The dashed blue line is calculated by spline interpolation of the BFIs measured with DCS across the 10-min interval starting 5 min before the injection. The filled green circle is the interpolated BFI at the ICG bolus injection time.

of direct absolute CBF calibration by applying this $\gamma$ coefficient to DCS-measured BFI from another group of brain-injured patients.

\subsection{Instrumentation}

Our custom-built instrument consists of a TR-NIRS module for measurement of tissue optical properties $\left(\mu_{a}\right.$ and $\left.\mu_{s}^{\prime}\right)$ and ICG tissue concentration, and a DCS module for measurement of CBF (i.e., BFI). The TR-NIRS light source is a commercial supercontinuum fiber laser (SuperK Extreme EXR-20, NKT Photonics Inc., Morganville, New Jersey) that emits short white-light pulses (400 to $2400 \mathrm{~nm}$, seed pulse width $\sim 5 \mathrm{ps}$ ) at a repetition rate of $78 \mathrm{MHz}$. The fiber laser was connected to an acousto-optic tunable filter (SuperK Cross, NKT Photonics Inc.) for programmable selection of specific wavelengths. The spectral width for $808 \mathrm{~nm}$ is $11 \mathrm{~nm}$, and the switching time between the wavelengths is $200 \mathrm{~ms}$. This light was delivered to tissue via a SuperK Connect fiber delivery system (FD7, NKT Photonics Inc.). Two hybrid single-photon sensitive photomultiplier tubes (PMA hybrid 50, Picoquant Photonics Inc., West Springfield, Massachusetts) were connected to a two-channel time-correlated single-photon counting module (HydraHarp 400, Picoquant Photonics Inc.) and were used for TR-NIRS photon time-of-flight measurements (1 ps resolution). The PMA hybrid detectors were equipped with electrically controlled shutters that were open only during TR-NIRS acquisition, and 665-nm long-pass colored glass filters (RG-665, Edmund Optics, Barrington, New Jersey) were installed to block visible room light. Ideally, a bandpass filter should be used to filter fluorescence from the excited ICG circulating in the brain tissue. ${ }^{44,54}$ However, due to the required switching between instrument operation modes, the fluorescence filter was not applied in this study. To ameliorate fluorescence effects, we calculated absorption changes from the integral of TPSF in Eq. (2) and we utilized carefully selected time-of-flight intervals ${ }^{44}$ wherein fluorescence contributions would be comparatively small.

The DCS light source is a continuous wave, long coherence length ( $\geq 8 \mathrm{~m}$ ) 785-nm diode laser (IBEAM-SMART-785-S-WS with Smartdock fiber coupler, Toptica Photonics Inc., Victor, New York) connected to a fiber-coupled electrically controlled 
shutter (OZ Optics, Ottawa, Ontario, Canada) for gated light delivery to tissue. DCS measurements of NIR light intensity correlations (10 Hz sampling rate) were made with four arrays of four high-sensitivity single-photon counting avalanche photodiodes (Excelitas SPCM-AQ4C, Pacer LLC, Palm Beach Gardens, Florida) connected to a multiple- $\tau$ 16-channel hardware correlator (Correlator.com, Bridgewater, New Jersey) operating in a burst mode. ${ }^{55}$ For more details about DCS and TR-NIRS instrumentation, we refer readers to recent reviews. $^{22,51,56,57}$

\subsubsection{Optical probe}

The optical probe used four 4-m long optical fiber bundles terminated with 3-mm right-angle prisms (MRA03-E03, Thorlabs, Newton, New Jersey) for tissue measurement (Fig. 3). The fiberoptic bundles were assembled by Fiberoptic Systems, Inc. (Simi Valley, California). Light emitted from the TR-NIRS and DCS lasers was delivered to the same location on the tissue via the source bundle [labeled a in Fig. 3(b)], i.e., a bundle of three graded index multimode fibers (62.5- $\mu \mathrm{m}$ core/0.275 NA; GIF625, Thorlabs); 1 for TR-NIRS, 1 for DCS, and 1 extra.

For the DCS long source-detector separation (SDS) measurement, light emerging from the tissue at a distance $r=2.5 \mathrm{~cm}$ from the source was detected with a bundle of 16 single-mode fibers [5- $\mu \mathrm{m}$ core/0.13 NA, $780 \mathrm{HP}$, Thorlabs, labeled c in Fig. 3(b)]; 15 of these fibers were connected to detection channels in the DCS instrument (1 fiber per channel). The 15 independent intensity autocorrelation functions acquired in parallel (at the "same" detector position) were subsequently averaged together to improve signal-tonoise ratio.

For the TR-NIRS long SDS measurement, light emerging from the tissue at a distance $r=3.2 \mathrm{~cm}$ from the source was detected with a bundle of 24 multimode fibers [200- $\mu \mathrm{m}$ core/ 0.22 NA, Fiberoptic Systems, labeled d in Fig. 3(b)] connected to a PMA hybrid detector. The optical probe also contained a hybrid TR-NIRS/DCS detection bundle [labeled b in Fig. 3(b)] for TR-NIRS and DCS measurement at $0.7-\mathrm{cm}$ SDS. Following a procedure described elsewhere, the optical fiber bundles were embedded at precise positions in urethane rubber. ${ }^{28}$ Note, a first-generation optical probe (rather than the standard probe) was employed for some of the measurements in one patient; in this patient, the source-detector distance may have varied slightly across monitoring days and would have contributed to larger-than-normal variations in calibration.

The optical probe was secured to the forehead superior to the frontal sinuses with sweat-resistant double-sided tape (in contact with skin: \#1522, 3M Health Care, St. Paul, Minnesota; in contact with probe: \#9917, 3M) on the bottom of the probe (four holes were cut in the tape at the four prism locations, such that the tape did not cover the prisms), and medical tape (Medipore Dress-It, \#2954, 3M) around the edges of the top of the probe. Further, a black cloth that spanned $\sim 2$ to $3 \mathrm{~cm}$ from the edges of the probe was taped down to block stray light.

In this paper, a semi-infinite tissue model of the head was utilized for the long TR-NIRS and DCS SDS measurements. The limitations of the semi-infinite model are explored in Sec. 4. (a)

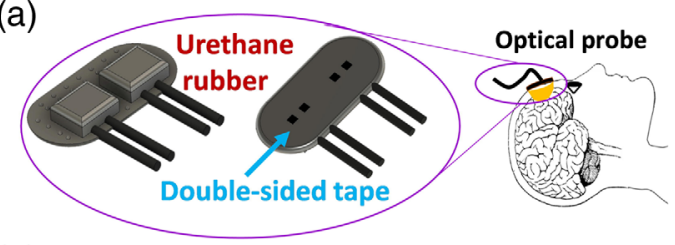

(b) Probe top view

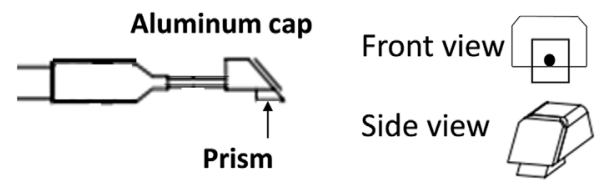

(c) TR-NIRS instrument response function measurement

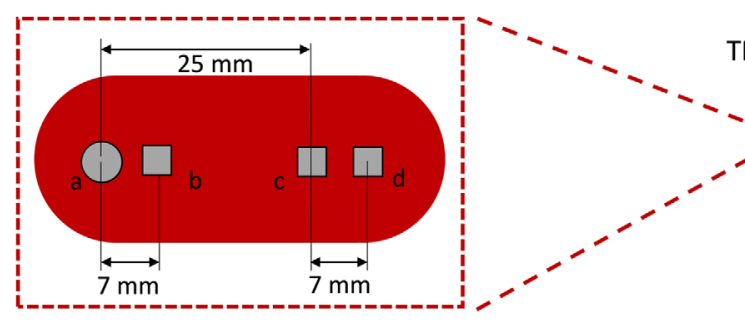

a. (3) graded index $62.5 \mu \mathrm{m}$ core/0.275NA

b. (2) $200-\mu m$ core/0.12 NA, (2) 5- $\mu m$ core/0.13NA; 2-mm black furcation tubing

c. (16) $5-\mu \mathrm{m}$ core/0.13NA; $3 \mathrm{~mm}$ black furcation tubing

d. (24) $200 \mu \mathrm{m}$ core/0.22NA; $3.8 \mathrm{~mm}$ black furcation tubing
R-NIRS source TR-NIRS detector

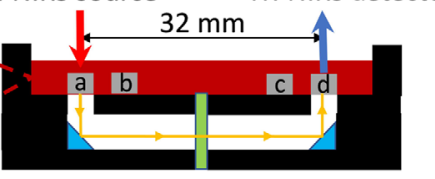

8.0 OD neutral density filter +1 ply kimwipe

3-mm right-angle prism mirror

- Light path, corners are right angles

Black holder: made of black delrin

Fig. 3 Schematic of the optical probe used for TR-NIRS measurement of tissue optical properties and DCS measurement of BFI (see main text for details). (a) The optical probe consists of prism-coupled fiber bundles embedded in urethane rubber (the prism-fiber connection is protected by an aluminum cap depicted on the right). It is secured to the forehead superior to the frontal sinuses with double-sided tape on the bottom and medical tape around the top (see Sec. 2.3.1). (b) The optical probe uses four fiberoptic bundles (labeled a, b, c, d) for TR-NIRS measurements at 0.7- and 3.2-cm SDS, and DCS measurements at $0.7-$ and $2.5-\mathrm{cm}$ SDS. In this paper, a semi-infinite tissue model of the head was utilized for the long TR-NIRS and DCS SDS measurements (see Secs. 2.1.1, 2.2, and 4). (c) Schematic of the TR-NIRS IRF measurement (side view; see Sec. 2.3.2). As the urethane rubber mold could not be bent 90 deg to place the source (position a) and detector (position d) prisms in direct contact, right-angle prism mirrors were used instead to direct the light from source to detector. 


\subsubsection{Instrument response function measurement}

The urethane rubber securing the prisms in place could not be bent to place the source (position a) and detector (position d) prisms in direct contact (Fig. 3). Therefore, the TR-NIRS IRF was measured using right-angle prism mirrors to direct the source light to the detector as shown in Fig. 3(c). To attenuate light properly and provide a wide angular distribution of the light, ${ }^{58}$ an 8.0 optical density (OD) neutral density filter (two sheets, 54-459, Edmund Optics) and one one-ply sheet of kimwipe (KW32, Thorlabs), i.e, as a diffuser, were placed in the middle of the light path. (Note, for fibers with larger NA, it is recommended that the diffuser be attached close to the tip of the detection fibers.) The FWHM bandwidth of the IRF was $190 \mathrm{ps}$ at $14 \mathrm{~mW}$ output power emerging from the source fiber (808-nm wavelength). The FWHM of the IRF is narrow compared with the typical width of the TPSF from brain tissue (i.e., FWHM $\approx 830$ ps at $3.2-\mathrm{cm}$ SDS). The IRF measurement was used to define the launch time of the incident source pulse in the calculation of the absorption change induced by ICG injection; it was also used to extract optical properties at $786 \mathrm{~nm}$ from the TR-NIRS measurement which, in turn, was used in the DCS fittings to derive DCS BFI.

\subsection{Monitoring Protocol}

Optical data acquisition was controlled with a custom-written software in the Labview environment (National Instruments, Austin, Texas). The instrument was used in two modes of operations (Fig. 4): (1) TR-NIRS measurement of absolute tissue optical absorption $\left(\mu_{a}\right)$ and tissue optical reduced scattering $\left(\mu_{s}^{\prime}\right)$ coefficients at six wavelengths $(730,750,786,810$, $830,850 \mathrm{~nm} ; 800-\mathrm{ms}$ exposure time per wavelength) with DCS measurement (10 s exposure time) of BFI and (2) ICG concentration measurement. In the first mode, the instrument sequentially interleaved TR-NIRS tissue measurements at six wavelengths with a DCS measurement (10 s exposure). This enabled us to derive $\mu_{a}$ and $\mu_{s}^{\prime}$, and BFI every $20 \mathrm{~s}$. The multispectral measurements of tissue optical absorption, which were made for the estimation of total hemoglobin concentration and tissue oxygen saturation, are not the focus of this paper. Only the optical properties from the 786-nm wavelength were used in this study to extract DCS BFI.
In the second mode, cerebral tissue ICG concentration and arterial ICG concentration were monitored with single-wavelength TR-NIRS ( $808 \mathrm{~nm}, 1 \mathrm{~Hz})$ and customized dye densitometry (see Sec. 6 and Appendix 1), respectively, to track the kinetics of ICG passage through the brain following intravenous bolus injection. ${ }^{19,20}$ A 2-min baseline measurement was made prior to ICG bolus injection, and 2-min continuous monitoring was carried out after the injection. After completion of this procedure, the instrument was switched back to the first operation mode. Two ICG bolus injections, spaced $4 \mathrm{~h}$ apart, were given per subject monitoring day.

\subsection{Patient Population and Study Design}

Seven adult patients in the neurointensive care unit were studied (six males, one female, age $43.9 \pm 13.5$ years). The enrolled patients were diagnosed with traumatic brain injury $(N=3)$, intracerebral hemorrhage $(N=2)$, or postischemic encephalopathy $(N=2)$. Patients were recruited through the Departments of Neurology and Anesthesiology and Critical Care with the Neurosurgery Clinical Research Division at the Hospital of the University of Pennsylvania. Written consent for all subjects was provided by legally authorized representatives, and all protocols/procedures were approved by the Institutional Review Board at the University of Pennsylvania, which adheres to the guidelines of the Common Rule and the Food and Drug Administration's Institutional Review Board's human subject regulations. Approximately four days of subject monitoring for eight hours on each day were targeted, though this goal was not always feasible due to clinical care needs.

On each subject monitoring day, the optical probe was secured above the fronto-parietal cortex (see Sec. 2.3.1) and the customized dye densitometer was secured to the subject's index finger (see Sec. 6 Appendix 1). Approximately $8 \mathrm{~h}$ of continuous optical measurements were acquired (as described in Sec. 2.4), and two intravenous ICG bolus injections ( 0.1 or $0.2 \mathrm{mg} / \mathrm{kg}$; <2 s per injection) were administered $\sim 4 \mathrm{~h}$ apart to calibrate the DCS BFI for absolute CBF measurement (see Fig. 5 for an example). ${ }^{19,20}$ Note, ICG is cleared from the body in $\sim 20 \mathrm{~min}$, and thus the calibration data could in principle have been taken more often. The 4-h time window we chose represents a compromise between the technical challenges of ICG injection, i.e., patient consent, ICG costs, and clinical

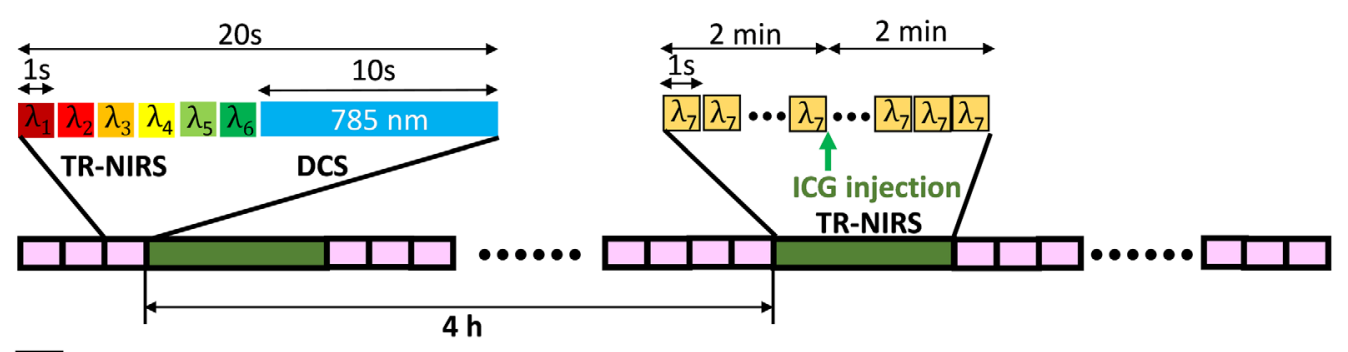

Mode 1, optical properties and BFI measurement

Mode 2, cerebral tissue ICG concentration measurement

$\lambda_{1}$ to $\lambda_{7}: 730,750,786,810,830,850,808 \mathrm{~nm}$

Fig. 4 Schematic of monitoring protocol (Sec. 2.4). During each monitoring day, TR-NIRS and DCS continuously measured tissue optical properties $\left(\mu_{a}\right.$ and $\left.\mu_{s}^{\prime}\right)$ and BFI (pink boxes, instrument mode 1). For the absolute CBF measurement via ICG bolus injection, continuous single-wavelength $(808 \mathrm{~nm})$ TR-NIRS measurements were made to track the kinetics of the ICG bolus through the TR-NIRS sampled cerebral tissue (green boxes, instrument mode 2). 

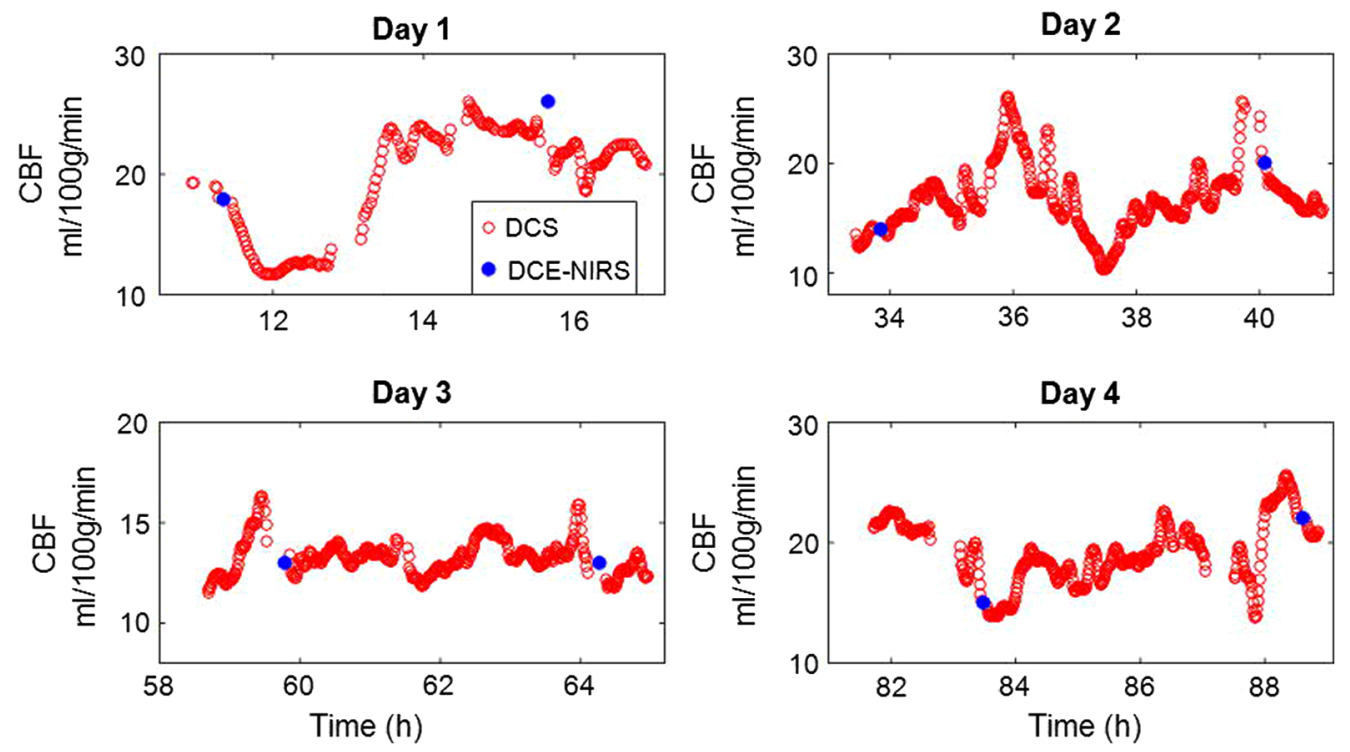

Fig. 5 Continuous calibrated absolute CBF measurements with DCS (red dots) and DCE-NIRS measurement of absolute CBF via intravenous ICG bolus injection (blue dots) in an exemplar patient diagnosed with traumatic brain injury. Here, the DCS measurements were calibrated with the first DCE-NIRS CBF measurement. This patient was continuously monitored for 4 days. For each monitoring day, two ICG bolus injections were administered $4 \mathrm{~h}$ apart.

personnel/workflow, against the need to generate adequate statistics to ascertain calibration. Thus, two calibration coefficients, $\gamma_{1}=\mathrm{CBF}_{0,1} / \mathrm{BFI}_{0,1}$ and $\gamma_{2}=\mathrm{CBF}_{0,2} / \mathrm{BFI}_{0,2}$, were obtained for a single patient across a single day. Here, the subscripts 1 and 2 denote first and second time points, respectively, of the two ICG injections. These calibration coefficients were compared to ascertain the stability of the ICG calibration technique according to Eq. (5). Note, due to clinical-care needs, for some of the monitoring days only 1 ICG injection was feasible.

We made 44 ICG injections across 24 monitoring days in the seven critically brain-injured patients. Of these 44 injections, absolute CBF could not be measured for eight of them; seven of these eight were due to the insufficient data quality in the $C_{a}(t)$ measurement, and one of the eight was due to significant heart rate increase during ICG injection (i.e., heart rate increased from 95 to $133 \mathrm{bpm}$ ) such that CBF was not constant during ICG bolus. Of the 24 monitoring days, continuous optical monitoring with two ICG boluses administered $4 \mathrm{~h}$ apart was feasible in 13 cases. Note, five of these monitoring days were for the cases wherein absolute CBF for one or two of the injections could not be assessed, for the reasons described above. For four of these monitoring days, it was only possible to give patients one ICG bolus. In the remaining 2 days, the optical probe needed to be removed for a clinical procedure.

\section{Results}

\subsection{DCS Single-Day Calibrations}

We calibrated DCS BF twice a day using the DCE-NIRS technique during the patient monitoring days wherein continuous BFI monitoring between doses was feasible $(N=13)$. Figure 6 shows comparison of two calibration coefficients, $\gamma_{1}$ and $\gamma_{2}$, for each patient across a single monitoring day. We observed a largely linear correlation $\left(R^{2}=0.80, N=23, P<0.01\right)$ and a slope close to unity (slope $=1.02 \pm 0.09$, here the error is the $95 \%$ confidence interval $[\mathrm{CI}]$ ) [Fig. 6(a)]. Additionally,
Bland-Altman analysis of these data [Fig. 6(b)] reveals a mean difference in the two calibration coefficients of $2.7 \times 10^{7}\left(\mathrm{~mL} \cdot 100 \mathrm{~g}^{-1} \cdot \mathrm{min}^{-1}\right) /\left(\mathrm{cm}^{2} / \mathrm{s}\right)$, which is not significantly different from zero $(p=0.67$ with Wilcoxon rank sum test). The limits of agreement between the two calibration coefficients [dashed lines in Fig. 6(b)], within which $95 \%$ of the differences reside are $4.7 \times 10^{8}$ and $-4.2 \times 10^{8}\left(\mathrm{~mL} \cdot 100 \mathrm{~g}^{-1} \cdot \mathrm{min}^{-1}\right) /\left(\mathrm{cm}^{2} / \mathrm{s}\right)$. This $95 \%$ CI $\left( \pm 4.4 \times 10^{8}\right)$ divided by the mean of the two calibration coefficients $\left[1.42 \times 10^{9}\left(\mathrm{~mL} \cdot 100 \mathrm{~g}^{-1} \cdot \mathrm{min}^{-1}\right) /\left(\mathrm{cm}^{2} / \mathrm{s}\right)\right]$ is $\pm 31 \%$. Thus, the DCE-NIRS technique for DCS BF calibration is quite stable across a single monitoring day for a single patient.

We used the coefficient of variation $(\mathrm{CV})$ to evaluate the stability of same-day calibration within a single subject. The CV across a single monitoring day was defined as $\left|\gamma_{2}-\gamma_{1}\right| / 0.5\left(\gamma_{2}+\gamma_{1}\right)$. Across 13 monitoring days, CV was $6.7 \pm 4.1 \%$ (mean $\pm \mathrm{SD}$ ). This result indicates that if each patient receives one-time DCS calibration for absolute CBF, then absolute $\mathrm{CBF}$ is expected to exhibit $\sim 7 \%$ variation after $\sim 4 \mathrm{~h}$.

\subsection{DCS Calibration across Multiple Days}

The absolute CBF measured with DCE-NIRS across all patients and all monitoring days was significantly correlated with the absolute BFI measured with DCS $\left[R^{2}=0.89, N=36\right.$, $P<0.01$ with $F$-test, see Fig. 7(a)]. Note, as DCE-NIRS and DCS sample similar brain volumes, and since both data analyses employed a semi-infinite head model, we forced the intercept to be zero in the linear regression analysis. We can derive a "best" DCS calibration coefficient from the slope $( \pm 95 \% \mathrm{CI})$ of the bestfit line, i.e., $\gamma=(1.24 \pm 0.08) \times 10^{9}(\mathrm{~mL} / 100 \mathrm{~g} / \mathrm{min}) /\left(\mathrm{cm}^{2} / \mathrm{s}\right)$. We then applied this estimate to all measurements in the same data to convert the DCS BFI to absolute $\mathrm{CBF}$, i.e., $\mathrm{CBF}_{\mathrm{DCS}}=$ $\gamma \times \mathrm{BFI}$, and we performed a Bland-Altman analysis to assess error in $\mathrm{CBF}_{\mathrm{DCS}}$. The Bland-Altman analysis reveals that a mean difference in CBF measured by two techniques, i.e, by 

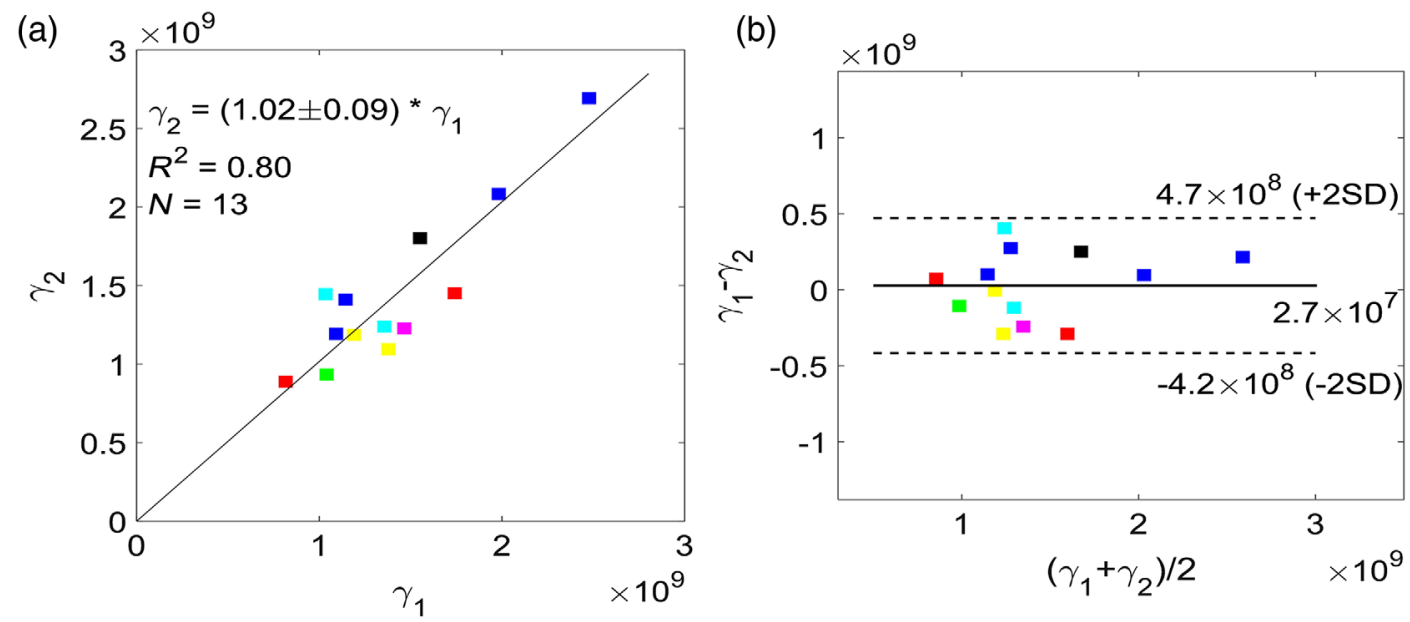

Fig. 6 (a) DCS calibration coefficients [unit: $\left(\mathrm{mL} \cdot 100 \mathrm{~g}^{-1} \cdot \mathrm{min}^{-1}\right) /\left(\mathrm{cm}^{2} / \mathrm{s}\right)$ ]from first ICG bolus injection (horizontal axis) and second injection (vertical axis) across $N=13$ monitoring days in 7 patients. Bolus injections are $4 \mathrm{~h}$ apart. The black line represents the best linear fit to the data with the intercept forced to be zero $\left(R^{2}=0.80\right.$, slope $\left.\pm 95 \mathrm{Cl}=1.02 \pm 0.09\right)$. (b) Bland-Altman plot of the difference in calibration coefficients from two ICG injections versus the mean of the two coefficients. The solid horizontal line indicates the mean difference between the two parameters computed across the study population, which is not significantly different from zero ( $p=0.67$ with Wilcoxon rank sum test). The dotted line indicates $95 \% \mathrm{Cl}$ limits for agreement. Different colors in (a) and (b) represent different patients.
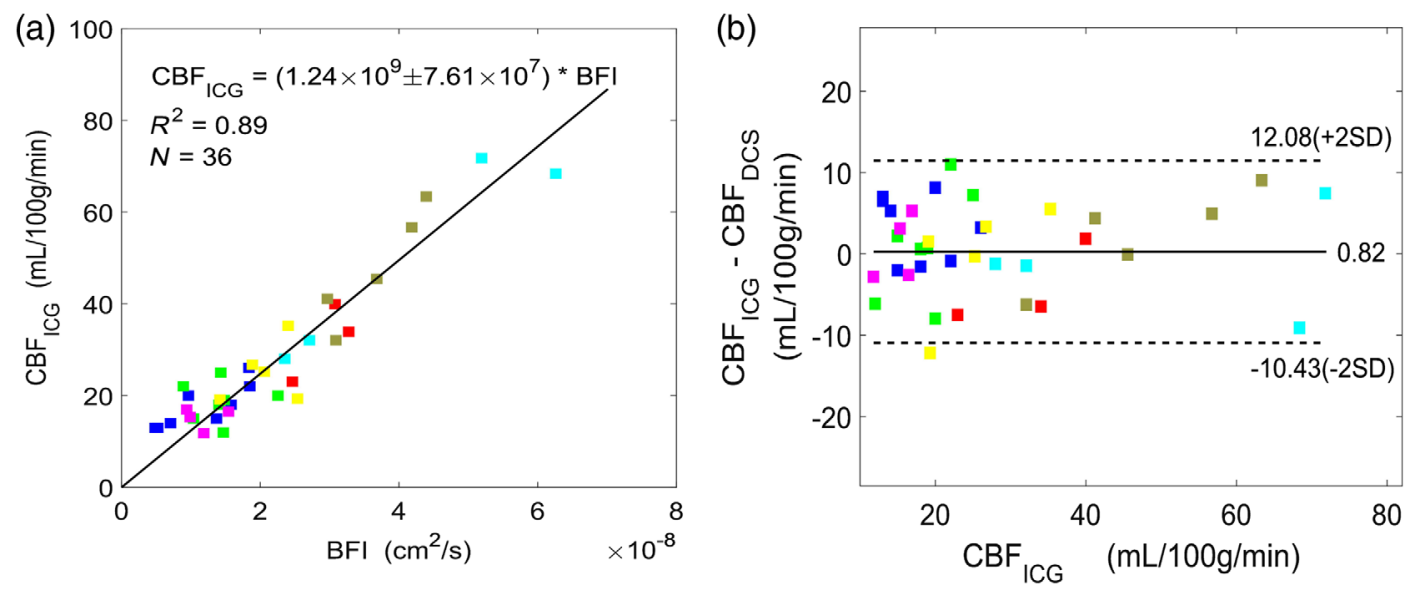

Fig. 7 (a) Absolute BF measured by DCE-NIRS technique (vertical axis) compared with the BFI measured with DCS (horizontal axis) across $N=36$ measurements. The black line represents the best linear fit to the data with the intercept forced to be zero (slope $=(1.24 \pm 0.08) \times 10^{9}, R^{2}=0.89, p<0.01$ with F-test). (b) Bland-Altman plot of the difference in absolute CBF measurements from DCS and DCE-NIRS techniques. The solid horizontal line indicates the mean difference between the two parameters computed across the study population, which is not significantly different from zero $(p=0.47$ with Wilcoxon rank sum test). The dotted line indicates $95 \% \mathrm{Cl}$ limits for agreement. Different colors in (a) and (b) represent different patients.

DCS and DCE-NIRS, of $0.82 \mathrm{~mL} / 100 \mathrm{~g} / \mathrm{min}$, is not significantly different from zero ( $p=0.47$ with Wilcoxon rank sum test) [Fig. 7(b)]. The limits of agreement between the two CBF measurement techniques [dashed lines in Fig. 7(b)], within which $95 \%$ of the differences resides, are -10.43 and $12.08\left(\mathrm{~mL} \cdot 100 \mathrm{~g}^{-1} \cdot \mathrm{min}^{-1}\right)$. The $95 \%$ CI [i.e., $\left.\pm 11\left(\mathrm{~mL} \cdot 100 \mathrm{~g}^{-1} \cdot \mathrm{min}^{-1}\right)\right]$ divided by the mean $\mathrm{CBF}_{\mathrm{ICG}}$ [i.e., $\left.29\left(\mathrm{~mL} \cdot 100 \mathrm{~g}^{-1} \cdot \mathrm{min}^{-1}\right)\right]$ is $\pm 38 \%$. Thus, a single $\mathrm{CBF}_{\mathrm{DCS}}$ measurement is expected to be within $38 \%$ of the actual CBF.

We used CV to evaluate the stability of calibration across multiple monitoring days within a single subject. Here, a single calibration coefficient was obtained for each monitoring day (i.e., the mean of $\gamma_{1}$ and $\gamma_{2}$, or just $\gamma_{1}$ if only one ICG injection was made). The $\mathrm{CV}$ coefficient for each patient is defined as the standard deviation of this calibration coefficient for each patient across multiple monitoring days divided by their mean. Across seven patients, $\mathrm{CV}$ was $21 \pm 14 \%$ (mean $\pm \mathrm{SD}$ ). This finding indicates that if each patient receives a one-time DCS calibration for absolute CBF monitoring, then absolute CBF is expected to exhibit $\sim 20 \%$ variation in the following days.

\subsection{Testing Calibrated-DCS for CBF of Brain-Injured Patients in Prior Study}

The strong linear relationship between CBF measured with DCE-NIRS and DCS BFI across multiple monitoring days 


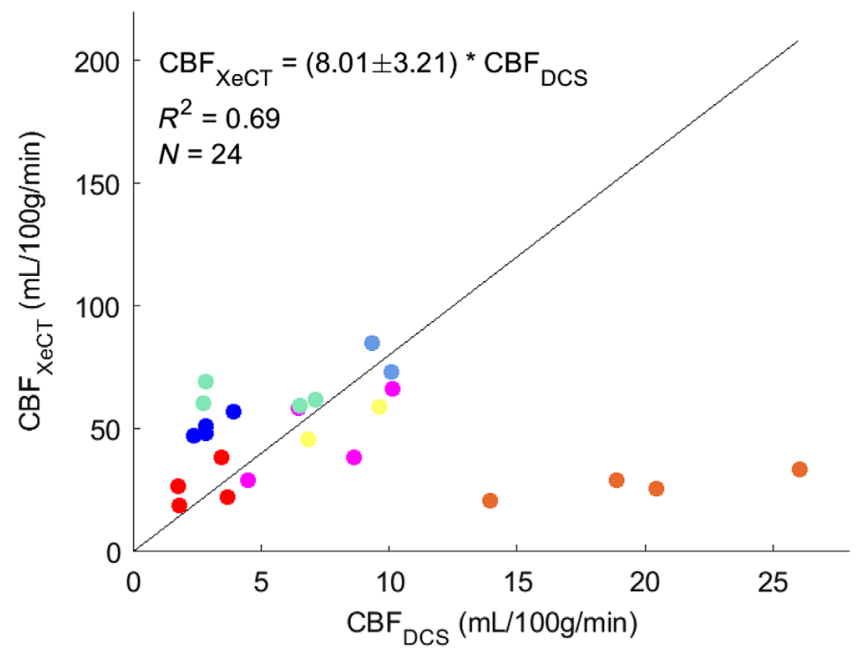

Fig. 8 Absolute CBF measured by XeCT technique (vertical axis) compared with the CBF measured with DCS (horizontal axis) across $N=24$ measurements. The black line represents the best linear fit to the data with the intercept forced to be zero (slope $=8.0 \pm 3.2$, $R^{2}=0.69, p<0.01$ with F-test). Different colors represent different patients.

(Fig. 7) suggests the potential for using DCS BFI directly as a measure of absolute $\mathrm{CBF}$, i.e., $\mathrm{CBF}_{\mathrm{DCS}}=\left(1.24 \times 10^{9}\right) \times$ BFI (see Fig. 7). We tested this approach in an independent dataset. Specifically, we compared $\mathrm{CBF}_{\mathrm{DCS}}$ obtained from previous DCS BFI measurements in seven brain-injured patients (i.e., carried out by our groups and published in Ref. 21) to concurrent measurements of absolute $\mathrm{CBF}$ made with the XeCT technique, i.e., $\mathrm{CBF}_{\mathrm{XeCT}}$. The previous study ${ }^{21}$ made BFI measurements on both sides (bilateral) of the forehead using a DCS SDS of $2.5 \mathrm{~cm}$ and employing a semi-infinite homogeneous tissue model for the head (i.e., the same theoretical model as used in the present paper). The $\mathrm{CBF}_{\mathrm{XeCT}}$ was obtained from averaging of the voxels on the cerebral cortex under the optical probes. ${ }^{21}$ During this prior study, pressors were administered to increase blood pressure, and a second concurrent set of bilateral $\mathrm{BFI}$ and $\mathrm{CBF}_{\mathrm{XeCT}}$ measurements was obtained for each patient. Thus, 24 concurrent $\mathrm{BFI}$ and $\mathrm{CBF}_{\mathrm{XeCT}}$ measurements were obtained across seven patients.

We converted the prior BFI data to $\mathrm{CBF}_{\mathrm{DCS}}$. A linear mixedeffects model was defined wherein $\mathrm{CBF}_{\mathrm{XeCT}}$ is the response variable, $\mathrm{CBF}_{\mathrm{DCS}}$ is the predictor variable, and patient ID is the grouping variable. The model was analyzed by the MATLAB $^{\circledR}$ function fitlme, and the results indicate that $\mathrm{CBF}_{\mathrm{XeCT}}$ was significantly correlated with the $\mathrm{CBF}_{\mathrm{DCS}}$ $\left(R^{2}=0.69, N=24, P<0.01\right.$ with $F$-test) (Fig. 8). From the slope $( \pm 95 \% \mathrm{CI})$ of a linear mixed-effect model, we estimate (8.0 \pm 3.2)-fold difference between $\mathrm{CBF}_{\mathrm{DCS}}$ converted from DCS BFI and $\mathrm{CBF}_{\mathrm{XeCT}}$, i.e., $\mathrm{CBF}_{\mathrm{XeCT}}=(8.0 \pm 3.2) \times$ $\mathrm{CBF}_{\mathrm{DCS}}$. These systematic differences between DCS-calibrated $\mathrm{CBF}$ and $\mathrm{CBF}_{\mathrm{XeCT}}$ can be understood, in part, to be a consequence of the semi-infinite model for brain (see below).

\section{Discussion}

To our knowledge, the present study is the first report of the calibration coefficient for absolute CBF monitoring with DCS in critically brain-injured adult patients. Several important previous studies have demonstrated that the same technique can be used to convert DCS data into absolute CBF on newborn piglets ${ }^{19}$ and babies. ${ }^{36}$ However, none have tested the stability of the calibration technique across long time periods. In this paper, we determine the stability of the calibration technique and investigate the utility of absolute CBF monitoring by direct DCS measurement throughout the course of treatment. Note, the instrumentation represents a state-of-the-art TR-NIRS and DCS system for various reasons, but such a complex TR-NIRS is not necessary for continuous absolute CBF monitoring.

In regard to stability, we found that the DCS calibration coefficient, $\gamma$, remains constant over at least $4 \mathrm{~h}$ of monitoring; good agreement was observed between the two daily calibration coefficients determined from two ICG bolus injections administered 4 hours apart [Fig. 6(a)]. Although only 4-h intervals were directly tested, $\gamma$ should remain stable for longer intervals, provided probe contact is stable. Intrasubject variation in $\gamma$ across multiple days, with probe removal and reapplication between daily monitoring sessions, was larger than the same-day results, with a mean coefficient of variation across subjects of $21 \% \pm 14 \%$ (Sec. 3.2). Thus, our results indicate that when removing the probe and reapplying the probe to roughly the same location the next day, a change in $\gamma$ of $\sim 20 \%$ can be expected (at least for the present protocols). Intrapatient variability, however, is sometimes observed to be larger across monitoring days. One large variation (marked with blue) was caused by use of first- and second generations of the probe (which varied slightly), and resultant variations in source-detector distance across monitoring days can introduce discrepancies into the calibration coefficients. In addition, some patients were observed to experience excessive sweating during the monitoring, requiring extra tape, and wrapping to secure the probe; this effect, in turn, could increase probe pressure and cause the calibration coefficient to vary. Generally, factors such as variation in the physiological state of underlying brain tissue, probe location, and probe pressure against the head can induce variation in the calibration coefficient. For this reason, our study utilized daily calibration, and the study suggests that daily calibration of DCS is optimal.

\subsection{Estimation of Absolute CBF from DCS BFI Measurements}

In regard to using the DCS BFI as a direct measure of absolute CBF, we observed a strong linear correlation $\left(R^{2}=0.89\right)$ between absolute CBF measured with DCE-NIRS and DCS BFI [Fig. 7(a)]. Using the slope of the best fit line as the $\gamma$ coefficient, estimation of absolute CBF from the DCS BFI is $\mathrm{CBF}_{\mathrm{DCS}}=\left(1.24 \times 10^{9}\right) \mathrm{BFI}$. Via Bland-Altman analysis, the mean difference between $\mathrm{CBF}_{\mathrm{DCS}}$ and $\mathrm{CBF}$ measured with DCE-NIRS, i.e., $\mathrm{CBF}_{\mathrm{ICG}}$, was not significantly different from zero $[p=0.47$ with Wilcoxon rank sum test, Fig. 7(b)]. The variability between $\mathrm{CBF}_{\mathrm{DCS}}$ and $\mathrm{CBF}_{\mathrm{ICG}}$, i.e., defined as the 95\% CI of their difference divided by the mean $\mathrm{CBF}_{\text {ICG }}$ across measurements, was $38 \%$. Thus, conservatively, our results suggest that $\mathrm{CBF}_{\mathrm{DCS}}$ should be within $40 \%$ of the $\mathrm{CBF}$, as measured by DCE-NIRS. Note that the DCS calibration coefficient of $\gamma=1.24 \times 10^{9}\left[\left(\mathrm{~mL} \cdot 100 \mathrm{~g}^{-1} \cdot \mathrm{min}^{-1}\right) /\left(\mathrm{cm}^{2} \cdot \mathrm{s}^{-1}\right)\right]$ obtained for this cohort is comparable with the DCS calibration coefficient previously obtained in piglets with the same DCENIRS technique, i.e., $1.14 \times 10^{9}\left[\left(\mathrm{~mL} \cdot 100 \mathrm{~g}^{-1} \cdot \mathrm{min}^{-1}\right) /\right.$ $\left.\left(\mathrm{cm}^{2} \cdot \mathrm{s}^{-1}\right)\right], R^{2}=0.89 .{ }^{19}$ 


\subsection{CBF Estimates Obtained with Semi-Infinite Head Model Underestimates True CBF}

We used the DCS calibration coefficient, $\gamma=1.24 \times$ $\left.\left.10^{9}\left[\mathrm{~mL} \cdot 100 \mathrm{~g}^{-1} \cdot \mathrm{min}^{-1}\right) /\left(\mathrm{cm}^{2} / \mathrm{s}\right)\right]\right)$, extracted from this cohort to convert prior BFI data in brain injured patients $(N=7)$ to absolute $\mathrm{CBF}$ (Sec. 3.3), which again, is denoted as $\mathrm{CBF}_{\mathrm{DCS}}$. In comparison with concurrent $\mathrm{CBF}$ measurements obtained with $\mathrm{XeCT}$, i.e., $\mathrm{CBF}_{\mathrm{XeCT}}$, linear regression analysis showed that although $\mathrm{CBF}_{\mathrm{DCS}}$ and $\mathrm{CBF}_{\mathrm{XeCT}}$ were correlated $\left(R^{2}=0.69\right), \mathrm{CBF}_{\mathrm{DCS}}$ underestimated $\mathrm{CBF}_{\mathrm{XeCT}}$ by roughly a factor of 8 (i.e., slope $\pm 95 \% \mathrm{CI}=8.0 \pm 3.2$ ). We can understand this systematic deviation (see below), but we also note that the early work had many limitations that could have weakened correlations. For example, the earlier paper assumed rather than measured tissue optical properties (i.e., $\mu_{a}=0.11 \mathrm{~cm}^{-1}$ and $\mu_{s}^{\prime}=12 \mathrm{~cm}^{-1}$ ) to compute BFI; this approach translates to errors in BFI. ${ }^{19,59,60}$ The overall underestimation, however, largely arises from using the semi-infinite homogeneous head model for determining $\gamma$ and BFI (Secs. 2.1 and 2.2).

It is known that the semi-infinite model underestimates localized CBF changes. In previous measurements of humans performing a finger tapping task, CBF relative changes computed with the semi-infinite model underestimated the true changes by a factor of $5{ }^{61}$ This result was subsequently confirmed in realistic simulations of the head. ${ }^{62}$ For absolute CBF estimates on healthy adult subjects with DCE-NIRS, mean CBF with the semi-infinite model was $8.3 \mathrm{~mL} /$ $100 \mathrm{~g} / \mathrm{min}$, which was believed to have large underestimation compared with values obtained by PET, i.e., a factor of $6 . .^{38,63,64}$ In two-layer simulations of the head, DCE-NIRS CBF measurements obtained from application of the semi-infinite model to continuous-wave NIRS data also underestimated the true CBF by around eightfold. ${ }^{63}$

To further elucidate this issue, we performed two-layer simulations of the head to assess underestimation of DCE-NIRS $\mathrm{CBF}$ measurements obtained from application of the semiinfinite model to simulated two-layer time-resolved NIRS data (details in Sec. 8 and Appendix 3). Overall, we found that the underestimation of $\mathrm{CBF}$ with the semi-infinite model ranged from 3.5 to 6.5 , with top layer thickness being the most sensitive parameter for different levels of underestimation (Sec. 8 and Appendix 3). Thus, the underestimation of $\mathrm{CBF}_{\mathrm{DCS}}$ compared with $\mathrm{CBF}$ measured from XeCT (slope $=8.0 \pm 3.2, N=24$, $R^{2}=0.69$, Sec. 3.3) partially overlapped with the range of underestimation found from this computer simulation.

\subsection{Limitations}

The use of the semi-infinite model for estimation of absolute CBF with DCE-NIRS is the most significant limitation for obtaining calibration coefficients in the present paper. Note, this limitation does not alter the paper's findings and conclusions about measurement stability. Different algorithms beyond the semi-infinite model have been proposed to improve the depth sensitivity of TR-NIRS measurements. ${ }^{20,65-69}$ One promising approach is the use of changes in the variance moment of the TPSF TR-NIRS measurement for retrieval of ICG concentration. ${ }^{68,70,71}$ The linearity between changes in the variance moment of TPSF and changes in absorption holds, however, only when the changes in absorption are sufficiently small. ${ }^{70,72}$ In simulations (see Sec. 4.2), we find that when the absorption changes are very large due to ICG, then a substantial underestimation of the cerebral absorption change is introduced when extracted by the variance moment analysis (e.g., by $\sim 72 \%$ ). To ameliorate this situation in the future, we will include higher order terms related to the absorption change; this method permits retrieval of more accurate absolute CBF and can be applied for bolus injection of ICG at high concentration. ${ }^{34}$ In a different vein, the short source-detector separation data were not used for several reasons. First, the TR-NIR measurement was not able to generate accurate optical properties, because the temporal width of IRF and tissue TPSF curves were too similar (FWHMs are 190 versus 300 ps). Also, we did not consistently obtain information about scalp and skull thickness (under the probe), because the necessary CT scans were not always taken during our optical monitoring session. Given these sources of error, we felt that only the large source-detector separations and semi-infinite head models were justified. In the future, we anticipate that short SDSs for both TR-NIRS and DCS measurement will be used along with a layered DCS model for better computation of cerebral BFI, ${ }^{28,73}$ and absolute DCS CBF calibration coefficient.

\section{Conclusion}

In this study, we demonstrated the long-term stability of noninvasive absolute CBF monitoring with DCE-NIRS calibrated DCS. The calibration was stable across a single monitoring day, and the variability for each patient across multiple monitoring days was moderate (20\% varying between 3\% and 40\%). The variability in calibration coefficient across multiple monitoring days and multiple subjects was small enough that a strong correlation between CBF measured with DCE-NIRS and DCS BFI was observed. However, for single measurements, deviations of up to $40 \%$ from the best fit line of DCE-NIRS CBF to DCS BFI can be an issue for some applications. If feasible, it is best to calibrate DCS with DCE-NIRS prior to every patient monitoring session. Finally, the semi-infinite model estimate of absolute CBF substantially underestimates the true CBF.

\section{Appendix 1: Principle of Customized ICG Dye Densitometer}

For measurement of arterial ICG concentration per volume of blood in the finger, i.e., $C_{\text {finger }}$ (Sec. 2.1), we used a customized dye densitometer that consisted of a two-wavelength (804 and $938 \mathrm{~nm}$ ) finger sensor (TL-301P, Nihon Kohden, Cambridge, Massachusetts) connected to an integrated analog front-end commercial pulse oximeter circuit board (AFE4400, Texas Instruments, Dallas, Texas), which was in turn connected to a laptop computer. The circuit board cyclically altered illumination of the finger between wavelengths $\lambda_{1}=804 \mathrm{~nm}$ and $\lambda_{2}=$ $938 \mathrm{~nm}$ (5-ms exposure per wavelength), and also recorded their temporal intensities transmitted through the finger, i.e., $\tilde{I}_{\left[\lambda_{1}\right]}(t)$ and $\tilde{I}_{\left[\lambda_{2}\right]}(t)$, at $100-\mathrm{Hz}$ sampling rate. Here, we introduce the principle of extracting $C_{\text {finger }}(t)$ from these measurements.

First, a finite impulse response digital bandpass filter with a passband frequency from $0.95 f_{h r}$ to $1.05 f_{h r}$, wherein $f_{h r}$ is the average heart rate across the four-minute interval encompassing the ICG injection (see Fig. 4, mode 2), was constructed in the MATLAB R2016a software environment (designfilt, Mathworks), and applied to $\tilde{I}_{\left[\lambda_{1}\right]}(t)$ and $\tilde{I}_{\left[\lambda_{2}\right]}(t)$. Hereafter, we will denote the bandpass filtered data as $I_{\left[\lambda_{1}\right]}(t)$ and $I_{\left[\lambda_{2}\right]}(t)$. The modified Beer-Lambert law $^{74,75}$ was next employed to relate the measured peak-to-peak amplitude of the pulsatile 
oscillation during the cardiac cycle in the bandpass filtered optical density to the peak-to-peak amplitudes of the pulsatile oscillations in light chromophore concentrations, i.e.,

$$
\begin{aligned}
\Delta \mathrm{OD}_{[\lambda]}(t) \equiv & -\log \left[\frac{I_{1[\lambda]}(t)}{I_{2[\lambda]}(t)}\right]=\left[\varepsilon_{H_{b} O[\lambda]} \Delta\left[H_{b} O\right](t)\right. \\
& \left.+\varepsilon_{H_{b}[\lambda]} \Delta\left[H_{b} R\right](t)+\varepsilon_{\mathrm{ICG}[\lambda]} \Delta[\mathrm{ICG}](t)\right] d_{[\lambda]},
\end{aligned}
$$

where $t$ refers to averaged times across single heart beats (e.g., for $\left.f_{h r}=60 \mathrm{bpm}, t=0.5 \mathrm{~s}, 1.5 \mathrm{~s}, 2.5 \mathrm{~s}, \ldots\right) ; I_{1[\lambda]}(t)$ and $I_{2[\lambda]}(t)$ are the peak and minimum bandpass filtered intensities at wavelength $\lambda$, respectively, during the cardiac cycle associated with the heart beat at time $t ; \Delta\left[H_{b} O\right](t), \Delta\left[H_{b} R\right](t)$, and $\Delta[\mathrm{ICG}](t)$ are the differences between the peak and minimum bandpass filtered oxyhemoglobin, deoxyhemoglobin, and ICG tissue concentrations (per volume of tissue), respectively, during the cardiac cycle at time $t ; \varepsilon_{H_{b} O[\lambda]}, \varepsilon_{H_{b \mid \lambda]}}$, and $\varepsilon_{\mathrm{ICG}[\lambda]}$ are the extinction coefficients of oxyhemoglobin, deoxyhemoglobin, and ICG (measured at $6.5 \mu \mathrm{M}$ concentration in plasma), respectively, at wavelength $\lambda_{;}^{.45}$ and $d_{[\lambda]}$ is the differential path length at wavelength $\lambda$. Assuming that heart rate induced blood volume oscillations are (1) faster than changes in chromophore concentrations (per volume blood) and (2) present only in the arterial compartment, then

$$
\begin{aligned}
\Delta\left[H_{b} O\right] & =S_{a} \Delta\left[H_{b} T\right], \\
\Delta\left[H_{b} R\right] & =\left(1-S_{a}\right) \Delta\left[H_{b} T\right], \\
\Delta[\mathrm{ICG}] & =C_{\text {finger }} \Delta\left[H_{b} T\right] /\left[H_{g} b\right],
\end{aligned}
$$

where $S_{a}$ is the arterial oxygen saturation, $\Delta\left[H_{b} T\right]$ is the difference between the peak and minimum bandpass filtered total hemoglobin concentration (per volume tissue), and $\left[H_{g} b\right]$ is the total hemoglobin concentration per volume blood. Note, the time-dependence $t$ is implicit for all parameters in Eq. (7). The expression for $\Delta[\mathrm{ICG}]$ in Eq. (7) is understood from noting that

$$
[\mathrm{ICG}] \equiv \frac{\mu_{\mathrm{mol}} \mathrm{ICG}}{V_{\text {tissue }}}=\frac{\mu_{\text {mol }} \mathrm{ICG}}{V_{\text {blood }}} \cdot \frac{V_{\text {blood }}}{V_{\text {tissue }}}=C_{\text {finger }} \cdot \frac{\left[H_{b} T\right]}{\left[H_{g} b\right]} .
$$

Substituting Eq. (7) into (6), we obtain

$$
\begin{aligned}
\Delta \mathrm{OD}_{[\lambda]}= & d_{[\lambda]}\left[\varepsilon_{H_{b} O[\lambda]} \cdot S_{a}+\varepsilon_{H_{b} R[\lambda]} \cdot\left(1-S_{a}\right)+\varepsilon_{\mathrm{ICG}[\lambda]} \frac{C_{\text {finger }}}{\left[H_{g} b\right]}\right] \\
& \cdot \Delta\left[H_{b} T\right] .
\end{aligned}
$$

Equation (8) is then used to generate a system of two equations that correspond to the two light wavelengths in the sensor $\left(\lambda_{1}=804 \mathrm{~nm}\right.$ and $\left.\lambda_{2}=938 \mathrm{~nm}\right)$, wherein $\varepsilon_{\mathrm{ICG}[\lambda 2]}=0$ :

$$
\begin{aligned}
\Delta \mathrm{OD}_{\left[\lambda_{1}\right]}= & d_{\left[\lambda_{1}\right]}\left[\varepsilon_{H_{b} O\left[\lambda_{1}\right]} \cdot S_{a}+\varepsilon_{H_{b} R\left[\lambda_{1}\right]} \cdot\left(1-S_{a}\right)\right. \\
& \left.+\varepsilon_{\mathrm{ICG}\left[\lambda_{1}\right]} \frac{\left.C_{\text {finger }}\right]}{\left[H_{g} b\right]}\right] \cdot \Delta\left[H_{b} T\right] \\
\Delta \mathrm{OD}_{\left[\lambda_{2}\right]}= & d_{\left[\lambda_{2}\right]}\left[\varepsilon_{H_{b} O\left[\lambda_{2}\right]} \cdot S_{a}+\varepsilon_{H_{b} R\left[\lambda_{2}\right]} \cdot\left(1-S_{a}\right)\right] \cdot \Delta\left[H_{b} T\right] .
\end{aligned}
$$

The ratio of Eqs. (9) and (10) is

$$
\begin{aligned}
\varphi & \equiv \frac{\Delta \mathrm{OD}_{\left[\lambda_{1}\right]}}{\Delta \mathrm{OD}_{\left[\lambda_{2}\right]}} \\
& =D \cdot \frac{\varepsilon_{H_{b} O\left[\lambda_{1}\right]} \cdot S_{a}+\varepsilon_{H_{b} R\left[\lambda_{1}\right]} \cdot\left(1-S_{a}\right)+\varepsilon_{\mathrm{ICG}\left[\lambda_{1}\right]} \cdot C_{\text {finger }} /\left[H_{\mathrm{g}} b\right]}{\varepsilon_{H_{b} O\left[\lambda_{2}\right]} \cdot S_{a}+\varepsilon_{H_{b} R\left[\lambda_{2}\right]} \cdot\left(1-S_{a}\right)},
\end{aligned}
$$

where $D \equiv d_{\left[\lambda_{1}\right]} / d_{\left[\lambda_{2}\right]} . \quad D$ is given by the rearrangement of Eq. (11) for the baseline time interval that spans the last $30 \mathrm{~s}$ prior to ICG injection ( $C_{\text {finger }, 0}=0$ at baseline):

$$
\begin{aligned}
D= & \varphi_{0}\left[\varepsilon_{H_{b} O\left[\lambda_{2}\right]} \cdot S_{a, 0}+\varepsilon_{H_{b} R\left[\lambda_{2}\right]} \cdot\left(1-S_{a, 0}\right)\right] /\left[\varepsilon_{H_{b} O\left[\lambda_{1}\right]}\right. \\
& \left.\cdot S_{a, 0}+\varepsilon_{H_{b} R\left[\lambda_{1}\right]} \cdot\left(1-S_{a, 0}\right)\right]
\end{aligned}
$$

where $\varphi_{0}$ and $S_{a, 0}$ denote the averages of $\varphi$ and $S_{a}$ across the baseline interval. Note, $S_{a, 0}$ is measured with a clinical pulse oximeter on the patient. Finally, with $D$ given by Eq. (12), Eqs. (9) and (10) can be directly solved for $C_{\text {finger }}$, i.e.,

$$
\begin{aligned}
C_{\text {finger }}= & \left\{\frac{\varphi}{D} \cdot\left[\varepsilon_{H_{b} O\left[\lambda_{2}\right]} \cdot S_{a, 0}+\varepsilon_{\mathrm{H}_{b} R\left[\lambda_{2}\right]} \cdot\left(1-S_{a, 0}\right)\right]\right. \\
& \left.-\varepsilon_{H_{b} O\left[\lambda_{1}\right]} \cdot S_{a, 0}-\varepsilon_{H_{b} R\left[\lambda_{1}\right]} \cdot\left(1-S_{a, 0}\right)\right\} \cdot \frac{\left[H_{\mathrm{g}} b\right]}{\varepsilon_{\mathrm{ICG}\left[\lambda_{1}\right]}},
\end{aligned}
$$

where the time-dependence $t$ is implicit for $C_{\text {finger }}$ and $\varphi$, and $S_{a}$ is approximated as a constant, i.e., $S_{a, 0}$, for the 4-min interval encompassing the ICG injection (see Fig. 4, mode 2) because the acute clinical pulse oximeter measurements following the ICG bolus injection are not reliable (the presence of ICG induces an artifactual drop in the clinical pulse oximeter measurement of $\left.S_{a}\right) .\left[H_{g} b\right]$ is also assumed to be constant, and its value is obtained from the closest clinical blood gas measurement to the ICG bolus injection. The $\left[H_{g} b\right]$ measurements were acquired within $12 \mathrm{~h}$ of the ICG bolus injections. Note, differences in $\left[H_{g} b\right]$ between the clinical blood gas measurement and ICG bolus injection timepoints are sources of errors in the calculation of $C_{\text {finger }}$.

\section{Appendix 2: Optical Properties Measured with TR-NIRS Based on Semi-infinite Medium}

In vivo cerebral tissue optical properties measurements at $\lambda=785 \mathrm{~nm}\left(\mu_{a}\right.$ and $\left.\mu_{s}^{\prime}\right)$ were obtained with TR-NIRS. Specifically, optical properties were extracted from the fits of TR-NIRS TPSF measurements to the time-domain analytical solution of the photon diffusion equation for the semi-infinite homogeneous medium and extrapolated zero boundary condition, ${ }^{76}$ i.e.,

$\phi(r, T)=0.118 \phi_{1}(r, T)+0.306 \phi_{2}(r, T)$,

where the solution, $\phi(r, T)$, is the sum of two terms that are proportional to the fluence rate, i.e., $\phi_{1}(r, T)$, and to the current or flux, i.e., $\phi_{2}(r, t)$, respectively. The proportionality coefficients, i.e., 0.118 and 0.306 , are based on a refractive index $n=1.4 ; T$ is the time-of-flight along the TPSF curve, and $r$ is the SDS. For semi-infinite media 


$$
\begin{aligned}
\phi_{1}(r, T)= & \frac{c}{(4 \pi D c T)^{3 / 2}} \exp \left(-\mu_{a} c T\right) \\
& \times\left\{\exp \left[-\frac{z_{0}^{2}+r^{2}}{4 D c T}\right]-\exp \left[-\frac{\left(z_{0}+2 z_{b}\right)^{2}+r^{2}}{4 D c T}\right]\right\},
\end{aligned}
$$

$$
\begin{aligned}
\Phi_{2}(r, T)= & \frac{1}{2} \cdot(4 \pi D c)^{-3 / 2} T^{-5 / 2} \exp \left(-\mu_{a} c T\right) \\
& \times\left[z_{0} \exp \left(-\frac{r_{1}^{2}}{4 D c T}\right)+\left(z_{0}+2 z_{b}\right) \exp \left(-\frac{r_{2}^{2}}{4 D c T}\right)\right]
\end{aligned}
$$

where $D, c, z_{0}, z_{b}, r_{1}$, and $r_{2}$ are defined below Eq. (4).

Due to the finite width of the laser pulse and its broadening by the instrumental response of the measuring system, the measured TPSF does not represent the true impulse of the tissue. To account for this, the theoretical curve generated by Eq. (14) is convolved with the IRF (Sec. 2.3) measured with the same fiber optics. ${ }^{58}$ Note that $T=\tilde{T}-t_{0}$, where $\tilde{T}$ is the time between the source trigger and photon detection, and $t_{0}$ is the launch time of the incident source pulse on the tissue (Sec. 2.1.1). A nonlinear optimization routine (MATLAB ${ }^{\circledR}$ function fminsearchbnd) was used to obtain $\mu_{a}, \mu_{s}^{\prime}$, and $t_{0}$ from the fit of the measured TPSF to the convolution of the IRF and theoretical TPSF $(\phi)$, i.e., $\mathrm{TPSF}_{\mathrm{fit}}=\mathrm{IRF} * \phi \quad\left(\mu_{a}\right.$ and $\mu_{s}^{\prime}$ constrained to be between 0.001 and $1 \mathrm{~cm}^{-1}$, and 0.1 and $50 \mathrm{~cm}^{-1}$, respectively; $t_{0}$ was constrained to be within $\pm 50 \mathrm{ps}$ of the time point when the IRF maximum occurs). Fitting is the process of minimizing a cost function between measured TPSF and the TPSF $F_{\text {fit }}$, where the measured TPSF and TPSF $F_{\text {fit }}$ were normalized to the area under the curve. The cost function was defined as the sum of the squared difference between measured TPSF and the TPSF $_{\text {fit }}$ scaled by squared root of measured TPSF at each time-of-flight. The fitting range was set to time of flights between the TPSF rising to $20 \%$ and falling to $10 \%$ of the maximal photon count.

\section{Appendix 3: Computer Simulation to Evaluate CBF Underestimation Using Semi-Infinite Model}

\begin{tabular}{|c|c|c|c|c|c|c|c|c|}
\hline & \multicolumn{5}{|c|}{ Physiology } & \multicolumn{2}{|c|}{$\begin{array}{l}\text { Optical properties } \\
(808 \mathrm{~nm})\end{array}$} & \multirow{2}{*}{$\begin{array}{c}\text { Anatomy } \\
\text { Layer Thickness }(\mathrm{cm})\end{array}$} \\
\hline & {$\left[\mathrm{H}_{\mathrm{b}} \mathrm{T}\right](\mu \mathrm{M})$} & $\mathrm{SO}_{2}(\%)$ & {$\left[\mathrm{H}_{\mathrm{g}} \mathrm{b}\right](\mathrm{g} / \mathrm{dL})$} & $\mathrm{BF}(\mathrm{mL} / 100 \mathrm{~g} / \mathrm{min})$ & Water Content (\%) & $\mu_{s}^{\prime}\left(\mathrm{cm}^{-1}\right)$ & $\mu_{a}\left(\mathrm{~cm}^{-1}\right)$ & \\
\hline Extracerebral & 42 & 65 & 10 & 10 & 75 & 12 & 0.09 & 1.2 \\
\hline Cerebral & 75 & 65 & 10 & 60 & 75 & 12 & 0.16 & Infinite \\
\hline
\end{tabular}

Computer simulation based on a two-layer model which mimics the extracerebral, i.e., scalp and skull, and cerebral tissues was

Table 1 Baseline physiological, anatomical, and optical parameters for the forward analytical solver.

(a)

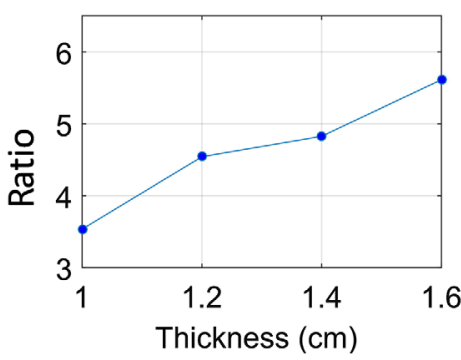

(e)

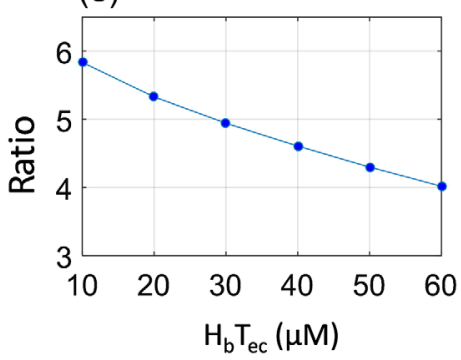

(b)

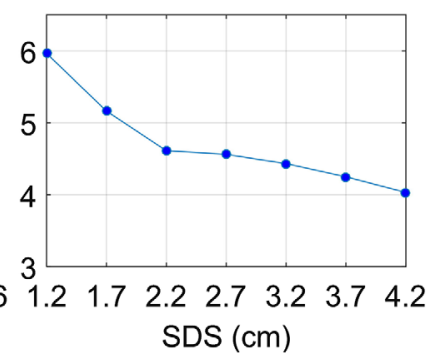

(f)

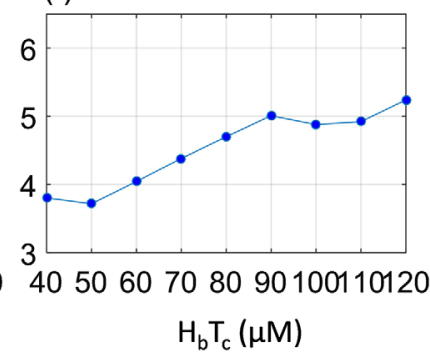

(c)

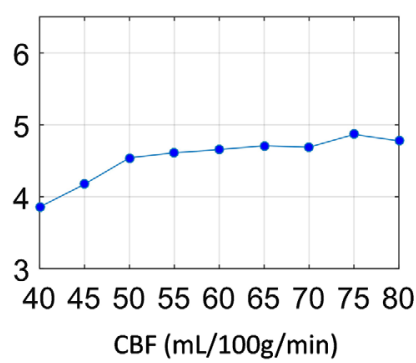

(g)

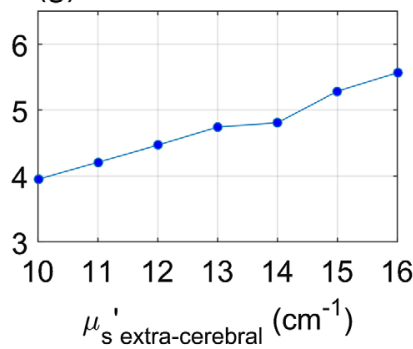

(d)

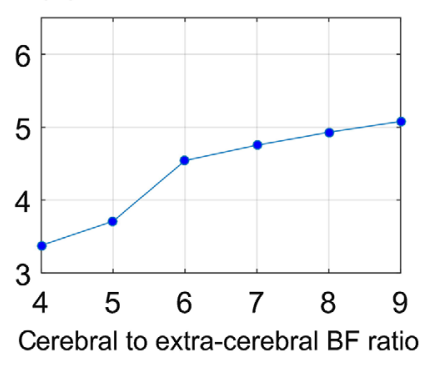

(h)

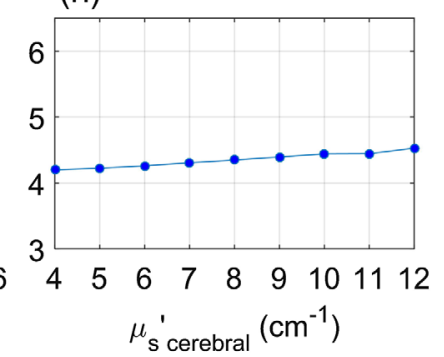

Fig. 9 Dependence of absolute CBF underestimation with semi-infinite model (i.e., actual CBF divided by the computed CBF with the semi-infinite model) on (a) extracerebral layer thickness, (b) source-detector separation for TR-NIRS measurement, (c) CBF, (d) cerebral to extra-CBF, (e) and (f) extracerebral and cerebral total hemoglobin concentration, and $(\mathrm{g})$ and $(\mathrm{h})$ extracerebral and cerebral tissue reduced scattering coefficients. 
performed to evaluate the underestimation in DCE-NIRS CBF measurements with the semi-infinite model. The passage of ICG bolus was simulated according to Eq. (1), where the arterial input function, $C_{a}(t)$, was obtained from a patient measurement with the ICG dose concentration at $0.2 \mathrm{mg} / \mathrm{kg}$. Note, Eq. (1) was used twice to compute $Q(t)$ for the extra-cerebral tissue [i.e., $Q_{e c}(t)$ ] and the cerebral tissue [i.e., $Q_{c}(t)$ ]. The impulse residue function, $R(t)$, was modeled as an exponential, i.e., $R_{c}(t)=\exp \left(-t / \mathrm{mtt}_{c}\right)$ and $R_{e c}(t)=\exp \left(-t / \mathrm{mtt}_{e c}\right)$ for the cerebral and extra-cerebral tissues, respectively. ${ }^{63}$ Here, $\mathrm{mtt}_{c}$ and $\mathrm{mtt}_{e c}$ are the mean transit times of blood through the DCE-NIRS sampled cerebral tissue volume and extracerebral tissue volume, respectively. These are estimated by $\mathrm{mtt}_{c}=\left[H_{b} T_{c}\right] /\left[H_{g} b\right]$ and $\mathrm{mtt}_{e c}=\left[H_{b} T_{e c}\right] /\left[H_{g} b\right]$, where $\left[H_{b} T_{c}\right]$ and $\left[H_{b} T_{e c}\right]$ are the total hemoglobin concentrations (per volume tissue) in cerebral and extra-cerebral tissues (e.g., measured with NIRS) and $\left[H_{g} b\right]$ is the total hemoglobin concentration (per volume blood) measured from blood gas, i.e., a blood sample is drawn by the clinician in the NICU and is analyzed by a spectrophotometric measurement in the clinic labs. The ICG concentration changes in the two layers were further converted to temporal absorption changes in the two layers by multiplying the extinction coefficient, $\varepsilon_{\mathrm{ICG}}$, at wavelength $808 \mathrm{~nm}$ according to Eq. (3). Temporal TPSF curves at certain SDS were generated with a two-layer forward solver (provided by Liemert and Kienle ${ }^{77}$ ) based on these temporal absorption changes at different layers and on constant cerebral and extra-cerebral tissue reduced scattering coefficients, tissue water content, and extra-cerebral layer thickness. Following the same method used to analyze the experiment data, the semi-infinite model was used to extract the absorption change based on the simulated TPSF curves according to Eq. (2), which was further converted to ICG concentrations. Deconvolution algorithm was then applied to the extract the absolute $\mathrm{CBF}$ values. The ratio between the simulated and extracted $\mathrm{CBF}$ values was taken to evaluate the underestimation induced by semi-infinite homogeneous model for a wide range of input parameters used to generate the simulated TPSF curves.

Specifically, the input parameters for a "baseline model" ${ }^{\prime 2,63,78}$ for computer simulation are set as shown in Table 1 with SDS at $3.2 \mathrm{~cm}$. Then, the input parameters were varied separately from the baseline model while all other input parameters were held constant (note, the baseline cerebral to extra-cerebral flow ratio of 6 was held constant instead of the absolute baseline extra-CBF). Variations in extra-cerebral layer thickness (from 1 to $1.6 \mathrm{~cm}$ ), SDS (from 1.2 to $4.2 \mathrm{~cm}$ ), CBF value (from 40 to $80 \mathrm{~mL} / 100 \mathrm{~g} / \mathrm{min}$ ), cerebral to extra-CBF ratio (from 4 to 9 via changing extra $\mathrm{CBF}$ ), total hemoglobin concentration in brain tissue (from 40 to $120 \mu \mathrm{M})$, total hemoglobin concentration in extra-cerebral tissues (from 10 to $60 \mu \mathrm{M}$ ), $\mu_{s}^{\prime}$ in extra-cerebral tissue (from 10 to $16 \mathrm{~cm}^{-1}$ ), ${ }^{78}$ and $\mu_{s}^{\prime}$ in cerebral tissue (from 4 to $12 \mathrm{~cm}^{-1}$ ) were tested. Figure 9 shows the dependence of the underestimation on different physiological, anatomical, and optical parameters as well as SDS. The range of underestimation varies from 3.5- to 6.0-fold, with extracerebral layer thickness being the most sensitive parameter.

\section{Disclosures}

No conflicts of interest, financial or otherwise, are declared by the authors.

\section{Acknowledgments}

We gratefully acknowledge Eileen Maloney, Elizabeth Mahanna, Suzanne Frangos, and Timothy Prior from Neurosurgery Clinical Research Division for clinical support. We thank Riley Wilson from 3M for sending us the double-sided tape. We thank Andre Liemert for providing the two-layer TR-NIRS forward solver. We acknowledge support from the National Institutes of Health (R01-NS082309-01A1, R01NS060653, and P41-EB015893).

\section{References}

1. C. Werner and K. Engelhard, "Pathophysiology of traumatic brain injury," Br. J. Anaesth. 99, 4-9 (2007).

2. W. Lewelt, L. W. Jenkins, and J. D. Miller, "Effects of experimental fluid-percussion injury of the brain on cerebrovascular reactivity of hypoxia and to hypercapnia," J. Neurosurg. 56, 332-338 (1982).

3. D. S. DeWitt, L. W. Jenkins, and D. S. Prough, "Enhanced vulnerability to secondary ischemic insults after experimental traumatic brain injury," New Horiz. 3, 376-383 (1995).

4. A. M. Kaufmann et al., "Ischemic core and penumbra in human stroke," Stroke 30, 93-99 (1999).

5. S. Aggarwal et al., "Cerebral hemodynamic and metabolic changes in fulminant hepatic failure: a retrospective study," Hepatology 19, 80-87 (1994).

6. S. Aggarwal et al., "Cerebral hemodynamic and metabolic profiles in fulminant hepatic failure: relationship to outcome," Liver Transpl. 11, 1353-1360 (2005).

7. S. Aggarwal et al., "Relationship of cerebral blood flow and cerebral swelling to outcome in patients with acute fulminant hepatic failure," Transpl. Proc. 23, 1978-1979 (1991).

8. A. R. Braun et al., "Regional cerebral blood flow throughout the sleepwake cycle. an H2(15)O PET study," Brain 120(Pt 7), 1173-1197 (1997).

9. D. S. Williams et al., "Magnetic resonance imaging of perfusion using spin inversion of arterial water," Proc. Natl. Acad. Sci. U. S. A. 89, 212-216 (1992).

10. W. D. Obrist et al., "Determination of regional cerebral blood flow by inhalation of 133-Xenon," Circ. Res. 20, 124-135 (1967).

11. H. Yonas et al., "Determination of irreversible ischemia by xenonenhanced computed tomographic monitoring of cerebral blood flow in patients with symptomatic vasospasm," Neurosurgery 24, 368-372 (1989).

12. D. Gur et al., "In vivo mapping of local cerebral blood flow by xenonenhanced computed tomography," Science 215, 1267-1268 (1982).

13. H. White and B. Venkatesh, "Applications of transcranial Doppler in the ICU: a review," Intensive Care Med. 32, 981-994 (2006).

14. W. A. Kofke et al., "Middle cerebral artery blood flow velocity and stable xenon-enhanced computed tomographic blood flow during balloon test occlusion of the internal carotid artery," Stroke 26, 1603-1606 (1995).

15. P. Vajkoczy et al., "Continuous monitoring of regional cerebral blood flow: experimental and clinical validation of a novel thermal diffusion microprobe," J. Neurosurg. 93, 265-274 (2000).

16. J. Cruz, "The first decade of continuous monitoring of jugular bulb oxyhemoglobinsaturation: management strategies and clinical outcome," Crit. Care Med. 26, 344-351 (1998).

17. K. G. Jordan, "Continuous EEG and evoked potential monitoring in the neuroscience intensive care unit," J. Clin. Neurophysiol. 10, 445-475 (1993).

18. T. M. Sundt, Jr. et al., "Cerebral blood flow measurements and electroencephalograms during carotid endarterectomy," J. Neurosurg. 41, 310-320 (1974)

19. M. Diop et al., "Calibration of diffuse correlation spectroscopy with a time-resolved near-infrared technique to yield absolute cerebral blood flow measurements," Biomed. Opt. Express 2, 2068-2081 (2011).

20. J. T. Elliott et al., "Quantifying cerebral blood flow in an adult pig ischemia model by a depth-resolved dynamic contrast-enhanced optical method," NeuroImage 94, 303-311 (2014). 
21. M. N. Kim et al., "Noninvasive measurement of cerebral blood flow and blood oxygenation using near-infrared and diffuse correlation spectroscopies in critically brain-injured adults," Neurocrit. Care 12, 173-180 (2010).

22. T. Durduran and A. G. Yodh, "Diffuse correlation spectroscopy for non-invasive, micro-vascular cerebral blood flow measurement," NeuroImage 85(Pt 1), 51-63 (2014).

23. R. C. Mesquita et al., "Direct measurement of tissue blood flow and metabolism with diffuse optics," Philos. Trans. A Math Phys. Eng. Sci. 369, 4390-4406 (2011).

24. T. Li et al., "Simultaneous measurement of deep tissue blood flow and oxygenation using noncontact diffuse correlation spectroscopy flowoximeter," Sci. Rep. 3, 1358 (2013).

25. S. A. Carp et al., "Combined multi-distance frequency domain and diffuse correlation spectroscopy system with simultaneous data acquisition and real-time analysis," Biomed. Opt. Express 8, 3993-4006 (2017).

26. K. Verdecchia et al., "Quantifying the cerebral metabolic rate of oxygen by combining diffuse correlation spectroscopy and time-resolved nearinfrared spectroscopy," J. Biomed. Opt. 18, 027007 (2013).

27. D. Wang et al., "Fast blood flow monitoring in deep tissues with realtime software correlators," Biomed. Opt. Express 7, 776-797 (2016).

28. W. B. Baker et al., "Pressure modulation algorithm to separate cerebral hemodynamic signals from extracerebral artifacts," Neurophotonics 2, 035004 (2015).

29. R. Cheng et al., "Noninvasive optical evaluation of spontaneous low frequency oscillations in cerebral hemodynamics," Neuroimage 62, 1445-1454 (2012).

30. N. Roche-Labarbe et al., "Near-infrared spectroscopy assessment of cerebral oxygen metabolism in the developing premature brain," J. Cereb. Blood Flow Metab. 32, 481-488 (2012).

31. L. X. Dong et al., "Diffuse optical measurements of head and neck tumor hemodynamics for early prediction of chemoradiation therapy outcomes," J. Biomed. Opt. 21, 085004 (2016).

32. D. W. Brown et al., "Quantitative near infrared spectroscopy measurement of cerebral hemodynamics in newborn piglets," Pediatr. Res. 51, 564-570 (2002).

33. K. St. Lawrence et al., "Measuring cerebral hemodynamics and energy metabolism by near-infrared spectroscopy," in Brain Energy Metabolism, J. Hirrlinger and H. S. Waagepetersen, Eds., pp. 265292, Springer, New York (2014).

34. D. Milej et al., "Quantification of cerebral blood flow in adults by dynamic contrast-enhanced NIRS: validation against MRI," in Optics and the Brain, Optical Society of America, p. BF2C.2 (2018).

35. K. M. Tichauer et al., "Near-infrared spectroscopy measurements of cerebral blood flow and oxygen consumption following hypoxiaischemia in newborn piglets," J. Appl. Physiol. (1985) 100, 850-857 (2006).

36. M. Diop et al., "Development of a combined broadband near-infrared and diffusion correlation system for monitoring cerebral blood flow and oxidative metabolism in preterm infants," Biomed. Opt. Express 6, 3907-3918 (2015).

37. R. Arora et al., "Preservation of the metabolic rate of oxygen in preterm infants during indomethacin therapy for closure of the ductus arteriosus," Pediatr. Res. 73, 713-718 (2013).

38. F. Gora et al., "Noninvasive measurement of cerebral blood flow in adults using near-infrared spectroscopy and indocyanine green: a pilot study," J. Neurosurg. Anesthesiol. 14, 218-222 (2002).

39. E. Keller et al., "Noninvasive measurement of regional cerebral blood flow and regional cerebral blood volume by near-infrared spectroscopy and indocyanine green dye dilution," Neuroimage 20, 828-839 (2003).

40. A. D. Edwards et al., "Cotside measurement of cerebral blood flow in ill newborn infants by near infrared spectroscopy," Lancet 332, 770-771 (1988).

41. J. Patel et al., "Measurement of cerebral blood flow in newborn infants using near infrared spectroscopy with indocyanine green," Pediatr. Res. 43, 34-39 (1998).

42. T. Iijima et al., "Cardiac output and circulating blood volume analysis by pulse dye-densitometry," J. Clin. Monit. 13, 81-89 (1997).

43. V. Ntziachristos et al., "Multichannel photon counting instrument for spatially resolved near infrared spectroscopy," Rev. Sci. Instrum. 70, 193-201 (1999).
44. O. Steinkellner et al., "Cerebral perfusion in acute stroke monitored by time-domain near-infrared reflectometry," Biocybern. Biomed. Eng. 32, 3-16 (2012).

45. S. Prahl, Optical absorption of indocyanine green (ICG) [online], 2018, http://omlc.ogi.edu/spectra/icg/index.html.

46. D. A. Boas, L. E. Campbell, and A. G. Yodh, "Scattering and imaging with diffusing temporal field correlations," Phys. Rev. Lett. 75, 1855-1858 (1995).

47. D. Boas and A. Yodh, "Spatially varying dynamical properties of turbid media probed with diffusing temporal light correlation," J. Opt. Soc. Am. A 14, 192-215 (1997).

48. M. Ninck, M. Untenberger, and T. Gisler, "Diffusing-wave spectroscopy with dynamic contrast variation: disentangling the effects of blood flow and extravascular tissue shearing on signals from deep tissue," Biomed. Opt. Express 1, 1502-1513 (2010).

49. T. Binzoni and F. Martelli, "Assessing the reliability of diffuse correlation spectroscopy models on noise-free analytical Monte Carlo data," Appl. Opt. 54, 5320-5326 (2015).

50. R. S. Chandran et al., "Detection and estimation of liquid flow through a pipe in a tissue-like object with ultrasound-assisted diffuse correlation spectroscopy," J. Opt. Soc. Am. A 32, 1888-1897 (2015).

51. Y. Shang, T. Li, and G. Yu, "Clinical applications of near-infrared diffuse correlation spectroscopy and tomography for tissue blood flow monitoring and imaging," Physiol. Meas. 38, R1 (2017).

52. L. He et al., "Using optical fibers with different modes to improve the signal-to-noise ratio of diffuse correlation spectroscopy flow-oximeter measurements," J. Biomed. Opt. 18, 037001 (2013).

53. D. A. Boas et al., "Establishing the diffuse correlation spectroscopy signal relationship with blood flow," Neurophotonics 3, 031412 (2016).

54. D. Milej et al., "Time-resolved detection of fluorescent light during inflow of ICG to the brain-a methodological study," Phys. Med. Biol. 57, 6725-6742 (2012).

55. G. Dietsche et al., "Fiber-based multispeckle detection for time-resolved diffusing-wave spectroscopy: characterization and application to blood flow detection in deep tissue," Appl. Opt. 46, 8506-8514 (2007).

56. A. Torricelli et al., "Time domain functional NIRS imaging for human brain mapping," NeuroImage 85, 28-50 (2014).

57. A. Pifferi et al., "New frontiers in time-domain diffuse optics, a review," J. Biomed. Opt. 21, 091310 (2016).

58. A. Liebert et al., "Fiber dispersion in time domain measurements compromising the accuracy of determination of optical properties of strongly scattering media," J. Biomed. Opt. 8, 512-516 (2003).

59. D. Irwin et al., "Influences of tissue absorption and scattering on diffuse correlation spectroscopy blood flow measurements," Biomed. Opt. Express 2, 1969-1985 (2011).

60. Z. Li et al., "Calibration of diffuse correlation spectroscopy blood flow index with venous-occlusion diffuse optical spectroscopy in skeletal muscle," J. Biomed. Opt. 20, 125005 (2015).

61. T. Durduran et al., "Diffuse optical measurement of blood flow, blood oxygenation, and metabolism in a human brain during sensorimotor cortex activation," Opt. Lett. 29, 1766-1768 (2004).

62. J. Selb et al., "Sensitivity of near-infrared spectroscopy and diffuse correlation spectroscopy to brain hemodynamics: simulations and experimental findings during hypercapnia," Neurophotonics 1, 015005 (2014).

63. T. S. Leung et al., "Theoretical investigation of measuring cerebral blood flow in the adult human head using bolus indocyanine green injection and near-infrared spectroscopy," Appl. Opt. 46, 1604-1614 (2007).

64. K. L. Leenders et al., "Cerebral blood flow, blood volume and oxygen utilization. Normal values and effect of age," Brain 113(Pt 1), 27-47 (1990).

65. A. Jelzow et al., "Separation of superficial and cerebral hemodynamics using a single distance time-domain NIRS measurement," Biomed. Opt. Express 5, 1465-1482 (2014).

66. F. Martelli et al., "Optimal estimation reconstruction of the optical properties of a two-layered tissue phantom from time-resolved singledistance measurements," J. Biomed. Opt. 20, 115001 (2015).

67. R. Re et al., "Effect of a thin superficial layer on the estimate of hemodynamic changes in a two-layer medium by time domain NIRS," Biomed. Opt. Express 7, 264-278 (2016). 
68. A. Liebert et al., "Bed-side assessment of cerebral perfusion in stroke patients based on optical monitoring of a dye bolus by time-resolved diffuse reflectance," NeuroImage 24, 426-435 (2005).

69. D. Milej et al., "Subtraction-based approach for enhancing the depth sensitivity of time-resolved NIRS," Biomed. Opt. Express 7, 4514-4526 (2016).

70. A. Liebert et al., "Time-resolved multidistance near-infrared spectroscopy of the adult head: intracerebral and extracerebral absorption changes from moments of distribution of times of flight of photons," Appl. Opt. 43, 3037-3047 (2004).

71. O. Steinkellner et al., "Optical bedside monitoring of cerebral perfusion: technological and methodological advances applied in a study on acute ischemic stroke," J. Biomed. Opt. 15, 061708 (2010).

72. W. B. Baker et al., "Modified Beer-Lambert law for blood flow," Biomed. Opt. Express 5, 4053-4075 (2014).

73. K. Verdecchia et al., "Assessment of the best flow model to characterize diffuse correlation spectroscopy data acquired directly on the brain," Biomed. Opt. Express 6, 4288-4301 (2015).

74. S. R. Arridge, M. Cope, and D. T. Delpy, "The theoretical basis for the determination of optical pathlengths in tissue: temporal and frequency analysis," Phys. Med. Biol. 37, 1531-1560 (1992).

75. D. T. Delpy et al., "Estimation of optical pathlength through tissue from direct time of flight measurement," Phys. Med. Biol. 33, 1433-1442 (1988).

76. A. Kienle and M. S. Patterson, "Improved solutions of the steady-state and the time-resolved diffusion equations for reflectance from a semiinfinite turbid medium," J. Opt. Soc. Am. A 14, 246-254 (1997).

77. A. Liemert and A. Kienle, "Application of the Laplace transform in time-domain optical spectroscopy and imaging," J. Biomed. Opt. 20, 110502 (2015).

78. A. Farina et al., "In-vivo multilaboratory investigation of the optical properties of the human head," Biomed. Opt. Express 6, 2609-2623 (2015).

Lian $\mathrm{He}$ is a postdoctoral researcher in physics and astronomy at the University of Pennsylvania. She graduated with a PhD in biomedical engineering at the University of Kentucky in August 2015. Her overall research goal is the development of optical technology for clinical biomedical applications, and her research interests include imaging and monitoring of cerebral blood flow and oxygen metabolism on NeurolCU patients.

Wesley B. Baker is a research associate in the division of neurology at the Children's Hospital of Philadelphia. As a physicist with specialization in optics in biological tissues, he is strongly interested in pursuing translational research that furthers the prognostic utility of optical tools in the treatment of brain injury. He graduated with a PhD in physics at the University of Pennsylvania in May 2015.

Daniel Milej is a postdoctoral fellow at Western University in London, Canada. He received his $\mathrm{PhD}$ in biomedical engineering from the Nalecz Institute of Biocybernetics and Biomedical Engineering, Polish Academy of Sciences in 2014. His current research focuses on designing, developing, and implementation of sophisticated optoelectronic medical and clinical instrumentation. He specifically focuses on brain imaging using time-domain diffuse optics and functional near-infrared spectroscopy.

Venkaiah C. Kavuri is a visiting scholar in physics and astronomy at the University of Pennsylvania. He was also a postdoc in physics and astronomy at the University of Pennsylvania from 2014 to 2016. He graduated with a $\mathrm{PhD}$ in biomedical engineering at the University of Texas Arlington in August 2014. His overall research goal is the development of hemoglobin-based optical techniques for noninvasive biomedical applications.

Rickson C. Mesquita is an assistant professor of physics at the University of Campinas (UNICAMP, São Paulo, Brazil). He leads a group in biomedical optics with interests focused on designing new instrumentation and developing innovative methods for diffuse optics in biological tissue. He has experience in translating diffuse optical techniques for clinical applications.

David R. Busch is an assistant professor in the Departments of Anesthesiology \& Pain Management and Neurology and Neurotherapeutics at the University of Texas Southwestern Medical Center. His work focuses on developing non- and minimally-invasive optical tools to monitor deep tissues in health and disease, as well as throughout therapy.

Kenneth Abramson is a research specialist in the Department of Physics and Astronomy at the University of Pennsylvania. His primary responsibilities include building and maintaining optical based instruments for clinical research including imaging, blood flow, and oxygen metabolism. He also designs and builds optical probes for instruments developed in the lab.

Jane Y. Jiang is an undergraduate student at Queens College, the City University at New York, double majoring in mathematics and physics. She was a summer research student at the Laboratory for Research on the Structure of Matter (LRSM) at the University of Pennsylvania in 2016.

Mamadou Diop is an assistant professor in the Department of Medical Biophysics at Western University and a research scientist at the Lawson Health Research Institute, both in London (Ontario, Canada). His research focuses on developing optical methods and systems for quantitative tissue imaging and sensing with applications in neuromonitoring, rheumatoid arthritis, breast cancer, wound healing, and nonhealing fractures.

Keith St. Lawrence is an associate professor in the Department of Medical Biophysics at Western University. His research focuses on developing quantitative methods of imaging cerebral blood flow and energy metabolism. This research encompasses a number of modalities, including diffuse optics, magnetic resonance imaging, and positron emission tomography.

Olivia Amendolia is a senior research nurse for the Neurosurgery Department of Clinical Research Division. She maintains regulatory compliance, coordinates study-specific procedures, and oversees the care of clinical trial patients. Her interests include improving outcomes in the acute care brain injured patient.

Francis Quattrone is a clinical research coordinator in the Department of Neurosurgery at the University of Pennsylvania. His primary responsibilities include clinical trial enrollment particular to the Neurological Intensive Care Unit for acute neurosurgical injuries and general inpatient trials in neurocritical care.

Ramani Balu is an assistant professor in the Department of Neurology at the University of Pennsylvania. In addition to providing clinical care for patients with life threatening brain injuries, he performs research evaluating the ability of novel monitoring tools to predict outcome and treatment response in brain injured patients.

W. Andrew Kofke is a professor in the Department of Anesthesiology and Critical Care at the University of Pennsylvania. He provides anesthesia and critical care support for patients with life threatening brain injuries and performs research evaluating the ability of novel monitoring tools to predict outcome and treatment response in brain injured and anesthetized patients.

Arjun G. Yodh is the James M. Skinner professor of science and the director of the Laboratory for Research on the Structure of Matter (LRSM) at the University of Pennsylvania. His current interests span fundamental and applied questions in condensed matter physics, medical and biophysics, and optical sciences. He has extensive experience utilizing diffuse optics techniques for monitoring and imaging of hemodynamics in living tissues. 